Bull. Soc. math. France

133 (2), 2005, p. 297-329

\title{
PROLONGEMENTS ANALYTIQUES D'UNE CLASSE DE FONCTIONS ZÊTA DES HAUTEURS ET APPLICATIONS
}

\author{
PAR D. ESSOUABRI
}

RÉSUMÉ. - Nous montrons dans la première partie l'existence d'un prolongement méromorphe à tout le plan complexe $\mathbb{C}$ et explicitons les propriétés et quelques conséquences, d'une large classe de séries zêta des hauteurs associées à l'espace projectif $\mathbb{P}_{n}(\mathbb{Q})(n \geq 1)$. Nous montrons dans la deuxième partie que, dans le cas du plan projectif éclaté en un point sur $\mathbb{Q}$, les fonctions zêta de hauteur associées aux fibrés en droite dont les classes sont à l'intérieur du cône des diviseurs effectifs possèdent des prolongements méromorphes à tout le plan complexe $\mathbb{C}$. Comme conséquence, ce résultat permet de redémontrer la conjecture de Manin dans ce cas mais avec un meilleur terme d'erreur que ceux connus. Il permet surtout de déterminer, le second terme en $\log B$ apparaissant dans la conjecture de Manin.

ABSTRACT (Analytic continuation of a class of zeta functions of heights)

In the first part of this paper, we prove that a large class of zeta functions associated to the projective space $\mathbb{P}_{n}(\mathbb{Q}),(n \geq 1)$, have meromorphic continuations to the whole complex plane $\mathbb{C}$ satisfying suitable properties and give some arithmetical consequences. In the second part, we prove that the height zeta functions associated to metrized line bundles on the projective plan blown up at a point, have meromorphic continuations to the whole complex plane $\mathbb{C}$ with moderate growth and give a set of candidate poles. As an application, we give a new proof of Manin's conjecture in this case, we calculate the second term and improve its error term.

Texte reçu le 13 mai 2003, accepté le 14 novembre 2003

D. Essouabri, Université de Caen, UFR des Sciences, Campus 2, Laboratoire de Mathématiques Nicolas Oresme (LMNO), CNRS UMR 6139, Bd Maréchal Juin, BP 5186, 14032 Caen (France) • E-mail : essoua@math.unicaen.fr

Classification mathématique par sujets (2000). — 14G05, 14G10, 14G40, $11 \mathrm{M} 41$.

Mots clefs. - Fonctions zêta des hauteurs, points rationnels, fibrés vectoriels, fonction de comptage, prolongement méromorphe, conjecture de Manin. 


\section{Introduction, description du problème et notations}

1.1. Introduction générale. - Soit $X$ une variété algébrique projective sur un corps de nombre $k$. On associe à tout fibré en droite $\mathcal{L}$ muni d'une métrique, une fonction hauteur $H_{\mathcal{L}}: X(k) \mapsto \mathbb{R}_{+}^{*}$ (voir [1], [7], [16], [14]). La conjecture de Manin raffinée par Peyre, Batyrev et Tschinkel, prédit que si la classe $L=[\mathcal{L}]$ de $\mathcal{L}$ dans $\operatorname{Pic}(X)$ est contenue dans l'intérieur du cône des diviseurs effectifs (i.e. le cône engendré par les classes des fibrés en droites $\mathcal{L}$ vérifiant $\Gamma(X, \mathcal{L}) \neq\{0\})$, il existe alors un ouvert Zariski dense $U$ de $X$ et des constantes $a(L), b(L)$ et $\theta(U, \mathcal{L})$ strictement positives, tels que lorsque $B$ tend vers $+\infty$,

$$
\begin{aligned}
N(U, \mathcal{L}, B): & =\left\{M \in X(k) \mid H_{\mathcal{L}}(M) \leq B\right\} \\
& =\frac{\theta(U, \mathcal{L}) B^{a(L)}(\log B)^{b(L)-1}}{a(L)(b(L)-1) !}(1+o(1)) .
\end{aligned}
$$

Même si cette conjecture est fausse en général (voir [2]), elle reste néanmoins pertinente pour une large classe de variétés et elle a été montrée dans certains cas (voir par exemple [3], [4], [5], [14], etc.).

La conjecture de Manin est liée via des théorèmes taubériens standard, aux propriétés analytiques des séries zêta des hauteurs

$$
Z_{U}(\mathcal{L} ; s)=\sum_{P \in U(k)} H_{\mathcal{L}}^{-s}(P) \quad(s \in \mathbb{C}) .
$$

Plus précisément, si on démontre que la fonction zêta $s \mapsto Z_{U}(\mathcal{L} ; s)$ possède un demi-plan de convergence et d'holomorphie de la forme $\left\{\operatorname{Re}(s)>\sigma_{a}\right\}$, qu'elle se prolonge méromorphiquement (avec croissance modérée sur les bandes verticales et $\sigma_{a}$ comme seul pôle) à un demi-plan de la forme $\left\{\operatorname{Re}(s)>\sigma_{a}-\delta\right\}$ $(\delta>0)$, les théorèmes taubériens standard impliquent alors qu'il existe un polynôme $Q \in \mathbb{R}[X] \backslash\{0\}$ et une constante $\delta^{\prime}=\delta^{\prime}(\delta, U, \mathcal{L})>0$ tels que

$$
N(U, \mathcal{L}, B)=\frac{\theta(U, \mathcal{L})}{a(L)(b(L)-1) !} B^{a(L)} Q(\log B)+\mathcal{O}\left(B^{a(L)-\delta^{\prime}}\right) \quad(B \rightarrow+\infty) .
$$

Il est à noter que $\delta^{\prime}$ est une fonction croissante en $\delta$. D'où l'intérêt d'avoir un prolongement méromorphe au plus grand demi-plan possible. À l'exception de certaines variétés de drapeaux généralisées (voir par exemple [7]) pour lesquelles le travail repose sur la difficile théorie de Langlands, les méthodes utilisées jusqu'à maintenant donnent le prolongement méromorphe à un demi-plan vertical mais pas à tout le plan complexe.

Nous montrons dans la première partie l'existence d'un prolongement méromorphe à tout le plan complexe $\mathbb{C}$ et explicitons les propriétés et quelques conséquences, d'une large classe de séries zêta des hauteurs associées à l'espace projectif $\mathbb{P}_{n}(\mathbb{Q})(n \geq 1)$ (voir la section qui suit pour les définitions de ces séries).

TOME $133-2005-\mathrm{N}^{\mathrm{O}} 2$ 
Nous montrons d'une façon simple dans la deuxième partie que, dans le cas du plan projectif éclaté en un point sur $\mathbb{Q}$, les fonctions zêta de hauteur associées aux fibrés en droite dont les classes sont à l'intérieur du cône des diviseurs effectifs possèdent des prolongements méromorphes à tout le plan complexe $\mathbb{C}$ et que ces prolongements sont à croissance modérée sur les bandes verticales. Ce résultat permet en particulier de redémontrer la conjecture de Manin dans ce cas mais avec un meilleur terme d'erreur que ceux connus. Notre travail permet aussi de déterminer dans ce cas, le second terme en $\log B$ apparaissant dans la conjecture de Manin; c'est la première fois que ce second terme est explicité.

Les deux hauteurs les plus connues sur $\mathbb{P}_{n}(\mathbb{Q})$ sont définies comme suit. Pour $M=\left(x_{0}: \ldots: x_{n}\right) \in \mathbb{P}_{n}(\mathbb{Q})$ avec $x_{i} \in \mathbb{Z}$ et $\operatorname{pgcd}\left(x_{0}, \ldots, x_{n}\right)=1$ (on peut toujours se ramener à ce cas), on définit (voir [14], [13], [16]) :

$$
H_{\infty}^{n}\left(x_{0}: \ldots: x_{n}\right)=\sup _{0 \leq i \leq n}\left|x_{i}\right| \text { et } H_{2}^{n}\left(x_{0}: \ldots: x_{n}\right)=\sqrt{x_{0}^{2}+\cdots+x_{n}^{2}} .
$$

Il est clair (voir ci-dessous par exemple) que si $X$ désigne une variété algébrique projective sur $\mathbb{Q}$, le choix d'une de ces deux hauteurs $H$ sur $\mathbb{P}_{n}(\mathbb{Q})$ détermine une famille de hauteurs $\left(H_{\mathcal{L}}\right)_{\mathcal{L}}(\mathcal{L}$ fibré en droite au-dessus de $X)$ sur $X(\mathbb{Q})$. Dans le cas du plan projectif éclaté en un point sur $\mathbb{Q}$, nous traitons par souci d'unité chacune des deux familles $\left(H_{\mathcal{L}}^{i}\right)_{\mathcal{L}}(i=\infty, 2)$ associées aux hauteurs classiques $H_{i}$ définies ci-dessus.

Il est à noter que dans le cas des espaces projectifs et du plan projectif éclaté en un point (comme dans la majorité des cas déjà résolus) les points rationnels sont paramétrisables et les résultats doivent être compris comme concernant les hauteurs associées à divers fibrés en droites $\mathcal{L}$.

Notons enfin que l'existence de prolongement méromorphe à tout le plan complexe d'une fonction zêta implique l'existence d'un développement limité de la fonction de comptage associée, mais il ne lui est pas équivalent. Il contient beaucoup plus d'informations arithmétiques qu'un simple développement limité. D'ailleurs même dans les cas où la conjecture est démontrée, l'existence d'un prolongement méromorphe n'est pas assurée comme le montre le contreexemple du plan projectif éclaté en deux points (voir [2]).

Remerciements. - Je tiens à remercier $\mathrm{Ph}$. Satgé pour ses conseils et suggestions le long de ce travail. Je remercie également D. Barlet, R. de la Bretèche et E. Peyre pour leurs critiques qui ont permis d'améliorer la présentation de la première version de cet article.

\subsection{Description du problème et notations}

1.2.1. Cas de l'espace projectif $\mathbb{P}_{n}(\mathbb{Q})(n \geq 1)$. - Soit $\mathbb{P}_{n}(\mathbb{Q})$ l'espace projectif de dimension $n$ sur $\mathbb{Q}$. Sur $\mathbb{P}_{n}(\mathbb{Q})$, on définit des hauteurs pour mesurer la taille des points. Les deux plus connues sont $H_{\infty}^{n}$ et $H_{2}^{n}$ définies par la relation (1). 
Plus généralement, on définit pour $P \in \mathbb{R}\left[X_{0}, \ldots, X_{n}\right]$ de degré $d \geq 1$, positif sur $\mathbb{R}_{+}^{n+1} \backslash\{(0, \ldots, 0)\}$, et pour tout $M=\left(x_{0}: \ldots: x_{n}\right) \in \mathbb{P}_{n}(\mathbb{Q})\left(x_{i} \in \mathbb{Z}\right)$ :

$$
H_{P}^{n}\left(x_{0}: \ldots: x_{n}\right)=P\left(\frac{\left|x_{0}\right|}{\operatorname{pgcd}\left(x_{0}, \ldots, x_{n}\right)}, \ldots, \frac{\left|x_{n}\right|}{\operatorname{pgcd}\left(x_{0}, \ldots, x_{n}\right)}\right)^{1 / d} .
$$

Avec ces notations, on a évidemment, $H_{2}=H_{P_{0}}$ où $P_{0}=X_{0}^{2}+\cdots+X_{n}^{2}$.

Dans la suite et sauf cas de confusion, on omettra l'exposant $n$ dans la définition des hauteurs sur $\mathbb{P}_{n}(\mathbb{Q})$, on notera $H_{i}$ la hauteur ${ }^{(1)} H_{i}^{n}(i=\infty, 2$ et $\left.P \in \mathbb{R}\left[X_{0}, \ldots, X_{n}\right]\right)$. On pose aussi

$$
V_{n}(\mathbb{Q})=\left\{M=\left(x_{0}: \ldots: x_{n}\right) \in \mathbb{P}_{n}(\mathbb{Q}) \mid x_{0} \ldots x_{n} \neq 0\right\} .
$$

On associe des fonctions zêta aux différentes hauteurs définies ci-dessus comme suit :

$$
\begin{cases}Z_{n, \infty}(s)=\sum_{M \in \mathbb{P}_{n}(\mathbb{Q})} \frac{1}{H_{\infty}(M)^{s}}, & Z_{n, \infty}^{*}(s)=\sum_{M \in V_{n}(\mathbb{Q})} \frac{1}{H_{\infty}(M)^{s}}, \\ Z_{n, 2}(s)=\sum_{M \in \mathbb{P}_{n}(\mathbb{Q})} \frac{1}{H_{2}(M)^{s}}, & Z_{n, 2}^{*}(s)=\sum_{M \in V_{n}(\mathbb{Q})} \frac{1}{H_{2}(M)^{s}} .\end{cases}
$$

Pour tout polynôme elliptique $P \in \mathbb{R}\left[X_{0}, \ldots, X_{n}\right]$ de degré $d \geq 1$ (voir la définition 1 ci-dessous), on pose

$$
Z_{\text {proj }}(P ; s)=\sum_{M \in \mathbb{P}_{n}(\mathbb{Q})} \frac{1}{H_{P}(M)^{s}}, \quad Z_{\text {proj }}^{*}(P ; s)=\sum_{M \in V_{n}(\mathbb{Q})} \frac{1}{H_{P}(M)^{s}} .
$$

En particulier,

$$
Z_{n, 2}(s)=Z_{\text {proj }}\left(P_{0} ; s\right) \quad \text { et } \quad Z_{n, 2}^{*}(s)=Z_{\text {proj }}^{*}\left(P_{0} ; s\right)
$$

où $P_{0}\left(x_{0}, \ldots, x_{n}\right)=\|x\|^{2}=x_{0}^{2}+\cdots+x_{n}^{2}$.

Dans la première partie de ce travail, nous montrons l'existence et donnons diverses propriétés des prolongements méromorphes à tout le plan complexe (et pas seulement à un demi-plan) de ces fonctions zêta de hauteurs. Comme application, nous obtenons à l'aide de théorèmes taubériens classiques des développements limités des fonctions de comptage

$$
\begin{aligned}
& N_{\mathbb{P}_{n}(\mathbb{Q})}(B)=\#\left\{M \in \mathbb{P}_{n}(\mathbb{Q}) \mid H(M) \leq B\right\}, \\
& N_{V_{n}(\mathbb{Q})}(B)=\#\left\{M \in V_{n}(\mathbb{Q}) \mid H(M) \leq B\right\}
\end{aligned}
$$

où $H$ est une des hauteurs définies ci-dessus. En particulier, on retrouve les déveoppements connus (voir [15], [16], [13]) dans les deux cas classiques $H=H_{\infty}$ et $H=H_{2}$.

(1) Cela ne définit une hauteur sur l'espace projectif au sens classique que si le polynôme est homogène. Il s'agit donc d'une extension de terminologie

TOME $133-2005-\mathrm{N}^{\mathrm{O}} 2$ 
1.2.2. Cas du plan projectif éclaté en un point. - Soit $X$ le plan projectif éclaté en un point $P_{0}$. On choisit les coordonnées de manière que

$$
\begin{aligned}
& P_{0}=(0: 0: 1), \\
& X(\mathbb{Q})=\left\{((x: y: z),(u: v)) \in \mathbb{P}_{2}(\mathbb{Q}) \times \mathbb{P}_{1}(\mathbb{Q}) \mid x v-y u=0\right\} .
\end{aligned}
$$

On note :

- $\pi: X \rightarrow \mathbb{P}_{2}$ la projection canonique,

- $E=\pi^{-1}\left(P_{0}\right)$ le diviseur exceptionnel,

- $\Lambda=\pi^{-1}(D)$ où $D$ est une droite projective quelconque de $\mathbb{P}_{2}$ ne contenant pas $P_{0}$.

On pose $U=X \backslash E$; donc $U$ est un ouvert Zariski dense de $X$. Avec ces notations le groupe de Picard de $X$ est donné par $\operatorname{Pic}(X)=\mathbb{Z} \Lambda \oplus \mathbb{Z} E$ et le cône effectif $C_{\text {eff }}(X) \subset \operatorname{Pic}(X) \otimes_{\mathbb{Z}} \mathbb{R}$ est donné par $C_{\text {eff }}(X)=\mathbb{R}_{+}(\Lambda-E) \oplus \mathbb{R}_{+} E$.

Soit $L_{a, b}=a \Lambda+b(\Lambda-E) \in \stackrel{\circ}{C}_{\text {eff }}(X)$, i.e. $a$ et $b$ sont deux entiers relatifs vérifiant $a>0$ et $a+b>0$. Pour tout $i=\infty, 2$ et avec les notations de la relation (1), la hauteur définie par

$$
H_{a, b}^{i}((x: y: z),(u: v))=\left(H_{i}^{2}((x: y: z))\right)^{a}\left(H_{i}^{1}((u: v))\right)^{b}
$$

correspond à une métrique adélique associée à $L_{a, b}$.

Le faisceau anticanonique $w_{X}^{-1}=3 \Lambda-E$ correspond à la hauteur $H_{2,1}^{i}$ et dans ce cas la conjecture de Manin prédit que

$$
a(L)=1 \quad \text { et } \quad b(L)=\operatorname{rang} \operatorname{Pic}(V)-1,
$$

résultat que nous reprouvons dans cet article.

\subsubsection{Notations et définition}

1) Dans toute la suite les symboles

$$
\begin{gathered}
f(\lambda, \boldsymbol{y}, \boldsymbol{x}) \ll_{\boldsymbol{y}} g(\boldsymbol{x}) \quad \text { uniformément en } \boldsymbol{x} \in X \text { et } \lambda \in \Lambda, \\
f(\lambda, \boldsymbol{y}, \boldsymbol{x})=\mathcal{O}_{\boldsymbol{y}}(g(\boldsymbol{x})) \quad \text { uniformément en } \boldsymbol{x} \in X \text { et } \lambda \in \Lambda
\end{gathered}
$$

ont le même sens et signifient qu'il existe $A=A(\boldsymbol{y})>0$, ne dépendant ni de $\boldsymbol{x}$ ni de $\lambda$, mais pouvant a priori dépendre des autres paramètres en particulier de $\boldsymbol{y}$, tels qu'on ait $|f(\lambda, \boldsymbol{y}, \mathbf{x})| \leq A g(\boldsymbol{x})$, pour tout $\boldsymbol{x} \in X$ et tout $\lambda \in \Lambda$. Quand il n'y a pas d'ambiguité, on omettra le mot uniformément dans ce qui précède.

2) Le symbole $f \asymp g$ signifie qu'on a à la fois $f \ll g$ et $g \ll f$.

3) On pose $n(x, y):=x / \operatorname{pgcd}(x, y)$ pour tout $(x, y) \in \mathbb{Z} \backslash\{(0,0)\}$.

4) $\|x\|=\sqrt{x_{1}^{2}+\cdots+x_{n}^{2}}$ désigne la norme euclidienne dans $\mathbb{R}^{n}$ pour tout $x=\left(x_{1}, \ldots, x_{n}\right) \in \mathbb{R}^{n}$.

5) On note $|\alpha|=\alpha_{1}+\cdots+\alpha_{n}$ et $\alpha$ ! $=\alpha_{1} ! \ldots \alpha_{n}$ ! pour tout $\alpha=$ $\left(\alpha_{1}, \ldots, \alpha_{n}\right) \in \mathbb{N}^{n}$.

6) On pose $\left(\begin{array}{l}s \\ k\end{array}\right)=s(s-1) \cdots(s-k+1) / k$ ! pour tout $s \in \mathbb{C}$ et tout $k \in \mathbb{N}$.

7) On note $s=\sigma+i \tau$ où $\sigma=\operatorname{Re}(s)$ et $\tau=\operatorname{Im}(s)$ pour $s \in \mathbb{C}$. 
8) On note $G(s)=\sum_{k, \ell \geq 1}\left(k^{2}+\ell^{2}\right)^{-s / 2}$ pour tout $s \in \mathbb{C}$ vérifiant $\operatorname{Re}(s)>2$. Nous étudierons en détail cette fonction dans le lemme 3.7.

9) On note $\mathcal{Z}(\zeta)=\{\beta \in \mathbb{C} \mid \zeta(\beta)=0\}$ où $\zeta$ désigne la fonction zêta de Riemann.

10) On note enfin $\Gamma$ (resp. $\gamma$ ) la fonction (resp. la constante) gamma d'Euler.

Nous donnons enfin la

DÉfinition 1. - Soient $P \in \mathbb{R}\left[X_{1}, \ldots, X_{n}\right]$ un polynôme de degré $d$ et $P_{d}$ sa partie homogène de degré $d$. On dit que $P$ est elliptique s'il vérifie

$$
\forall x \in \mathbb{R}_{+}^{n} \backslash\{(0, \ldots, 0)\}, \quad P_{d}(x)>0 \text { et } P(x)>0 .
$$

\section{2. Énoncés des principaux résultats}

\section{1. Énoncés dans le cas de l'espace projectif $\mathbb{P}_{n}(\mathbb{Q})$}

Proposition 2.1. - 1) Les fonctions zêta

$$
s \mapsto Z_{n, \infty}(s)=\sum_{M \in \mathbb{P}_{n}(\mathbb{Q})} \frac{1}{H_{\infty}(M)^{s}} \quad \text { et } \quad s \mapsto Z_{n, \infty}^{*}(s)=\sum_{M \in V_{n}(\mathbb{Q})} \frac{1}{H_{\infty}(M)^{s}}
$$

convergent absolument et sont holomorphes dans le demi-plan $\{\operatorname{Re}(s)>n+1\}$ et y vérifient

$$
\begin{aligned}
& Z_{n, \infty}(s)=\frac{1}{\zeta(s)} \sum_{\substack{\ell \in\{0, \ldots, n\} \\
\ell \neq n-1}} a(\ell, n) \zeta(s-\ell), \\
& Z_{n, \infty}^{*}(s)=\frac{2^{n}}{\zeta(s)} \sum_{\ell=0}^{n}(-1)^{n-\ell}\left(\begin{array}{c}
n+1 \\
\ell
\end{array}\right) \zeta(s-\ell)
\end{aligned}
$$

où $\zeta$ est la fonction zêta de Riemann et où $a(\ell, n)=\sum_{r=\ell}^{n}\left(\begin{array}{c}n+1 \\ r+1\end{array}\right)\left(\begin{array}{c}r+1 \\ \ell\end{array}\right) 2^{r}(-1)^{r-\ell}$ pour tout $\ell=0, \ldots, n$.

2) En particulier, $s \mapsto Z_{n, \infty}(s)$ (resp. $s \mapsto Z_{n, \infty}^{*}(s)$ ) possède un prolongement méromorphe à tout le plan complexe $\mathbb{C}$ avec des pôles contenus dans l'ensemble

$$
S_{n}=(\{j \in \mathbb{N} \mid 2 \leq j \leq n+1\} \backslash\{n\}) \cup\{\beta \in \mathbb{C} \mid \zeta(\beta)=0\}
$$

(resp. $\left.S_{n}^{*}=S_{n} \cup\{n\}\right)$.

3) En outre, $s=n+1$ est l'abscisse de convergence des deux séries $Z_{n, \infty}(s)$ et $Z_{n, \infty}^{*}(s)$ et c'est un pôle simple de résidu $2^{n}(n+1) / \zeta(n+1)$ pour ces deux fonctions. 
Corollaire 2.2. - Pour tout $k \in \mathbb{N}^{*}$, on a

$$
\begin{aligned}
g(k) & :=\#\left\{M \in \mathbb{P}_{n}(\mathbb{Q}) \mid H_{\infty}(M)=k\right\}=\sum_{\substack{\ell=0 \\
\ell \neq n-1}}^{n} a(\ell, n) k^{\ell}\left(\sum_{d \mid k} \frac{\mu(d)}{d^{\ell}}\right), \\
g^{*}(k) & :=\#\left\{M \in V_{n}(\mathbb{Q}) \mid H_{\infty}(M)=k\right\}=2^{n} \sum_{\ell=0}^{n}(-1)^{n-\ell}\left(\begin{array}{c}
n+1 \\
\ell
\end{array}\right) k^{\ell}\left(\sum_{d \mid k} \frac{\mu(d)}{d^{\ell}}\right) .
\end{aligned}
$$

REMARQUe. - La proposition 2.1 permet de retrouver facilement, via un argument taubérien, les estimations très classiques suivantes (voir [14], [13]) :

$$
\begin{aligned}
& N_{n}(B)=\#\left\{M \in \mathbb{P}_{n}(\mathbb{Q}) \mid H_{\infty}(M) \leq B\right\}=\frac{2^{n} B^{n+1}}{\zeta(n+1)}+\mathcal{O}\left(\varepsilon_{n}(B)\right), \\
& N_{n}^{*}(B)=\#\left\{M \in V_{n}(\mathbb{Q}) \mid H_{\infty}(M) \leq B\right\}=\frac{2^{n} B^{n+1}}{\zeta(n+1)}+\mathcal{O}\left(\varepsilon_{n}(B)\right)
\end{aligned}
$$

où $\varepsilon_{n}(B)=B^{n}$ si $n \geq 2$ et $\varepsilon_{n}(B)=B \log B$ si $n=1$.

ThÉORÈme 2.3. - Soit $P \in \mathbb{R}\left[X_{0}, \ldots, X_{n}\right]$ un polynôme elliptique. Alors :

1) Les fonctions zêta

$$
s \longmapsto Z_{\text {proj }}(P ; s)=\sum_{M \in \mathbb{P}_{n}(\mathbb{Q})} \frac{1}{H_{P}(M)^{s}}, s \longmapsto Z_{\text {proj }}^{*}(P ; s)=\sum_{M \in V_{n}(\mathbb{Q})} \frac{1}{H_{P}(M)^{s}}
$$

convergent absolument et sont holomorphes dans $\{\operatorname{Re}(s)>n+1\}$. De plus, elles possèdent des prolongements méromorphes à tout le plan complexe avec des pôles contenus dans l'ensemble

$$
S(P)=\{n+1-k \mid k \in \mathbb{N}\} \cup\{\beta-k \mid \zeta(\beta)=0 \text { et } k \in \mathbb{N}\} .
$$

2) En outre, $\sigma_{0}=n+1$ est l'abscisse de convergence de $Z_{\text {proj }}(P ; s)$ et de $Z_{\text {proj }}^{*}(P ; s)$; c'est un pôle simple pour ces deux fonctions de résidus

$$
\operatorname{Res}_{s=n+1} Z_{\text {proj }}(P ; s)=\operatorname{Res}_{s=n+1} Z_{\text {proj }}^{*}(P ; s)=\frac{2^{n}}{\zeta(n+1)} \int_{\mathbb{S}^{n} \cap \mathbb{R}_{+}^{n+1}} P_{d}^{-(n+1) / d}(u) \mathrm{d} \sigma(u)
$$

où $\mathbb{S}^{n}$ est la sphère unité de $\mathbb{R}^{n+1}$ et $\mathrm{d} \sigma$ la mesure de Lebesgue sur cette sphère.

3) De plus, $1,2, \ldots, n+1$ sont les seuls pôles éventuels de $Z_{\text {proj }}$ et de $Z_{\text {proj }}^{*}$ dans le demi-plan $\{\operatorname{Re}(s) \geq 1\}$ et sont au plus simples.

4) Pour tout $\varepsilon>0$, on a

$$
\left|Z_{\text {proj }}(P ; s)\right|+\left|Z_{\text {proj }}^{*}(P ; s)\right| \ll_{\sigma, \varepsilon} 1+|\tau|^{n+1-\sigma+\varepsilon}
$$

uniformément en $s=\sigma+i \tau \in \mathbb{C}$ vérifiant $\sigma>1$ et $|\tau| \geq 1$.

BULLETIN DE LA SOCIÉTÉ MATHÉMATIQUE DE FRANCE 
Corollaire 2.4. - Soit $P \in \mathbb{R}\left[X_{0}, \ldots, X_{n}\right]$ un polynôme elliptique. Alors pour tout $\varepsilon>0$ et $B \rightarrow+\infty$, on a

$$
\begin{aligned}
& N_{\text {proj }}(P ; B)=\#\left\{M \in \mathbb{P}_{n}(\mathbb{Q}) \mid H_{P}(M) \leq B\right\}=c B^{n+1}+\mathcal{O}_{\varepsilon}\left(B^{n+\delta_{n}+\varepsilon}\right), \\
& \quad N_{\text {proj }}^{*}(P ; B)=\#\left\{M \in V_{n}(\mathbb{Q}) \mid H_{P}(M) \leq B\right\}=c B^{n+1}+\mathcal{O}_{\varepsilon}\left(B^{n+\delta_{n}+\varepsilon}\right), \\
& \text { où } c=\left(2^{n} /(n+1) \zeta(n+1)\right) \int_{\mathbb{S}^{n} \cap \mathbb{R}_{+}^{n+1}} P_{d}^{-(n+1) / d}(u) \mathrm{d} \sigma(u), \text { où } \delta_{n}=0 \text { si } P \text { est } \\
& \text { homogène et } \delta_{n}=1 / n \text { sinon. }
\end{aligned}
$$

REMARQue. - La constante $c$ du corollaire 2.4 cö̈ncide avec celle connue dans le cas des polynômes homogènes (voir [14], [13]).

2.2. Énoncés des résultats dans le cas plan projectif éclaté en un point. - On utilisera dans cette partie les notations des paragraphes 1.2.2 et 1.2.3. En particulier, $X$ désigne le plan projectif éclaté en un point et $U=X \backslash E$ où $E$ est le diviseur exceptionnel (voir $\S 1.2 .2$ ).

Soient $a$ et $b$ deux entiers relatifs fixés et vérifiant $a>0$ et $a+b>0$ et $\mathcal{L}_{a, b}$ le diviseur associé. Les conditions sur $a$ et $b$ sont équivalentes au fait que la classe de $\mathcal{L}_{a, b}$ est à l'intérieur du cône effectif. Soient

$$
\alpha=\sup \left(\frac{2}{a}, \frac{3}{a+b}\right), \quad \alpha^{\prime}=\sup \left(\left\{\frac{2}{a}, \frac{3}{a+b}, \frac{2}{a+b}, \frac{1}{a}\right\} \backslash\{\alpha\}\right)<\alpha .
$$

Remarquons d'abord que si $b \leq 0$ (i.e. $\mathcal{L}_{a, b}$ n'est pas ample), alors pour tout $i \in\{\infty, 2\}$ et tout $B>1$, on a

$$
N_{i}\left(E(\mathbb{Q}), \mathcal{L}_{a, b}, B\right)=\#\left\{(u: v) \in \mathbb{P}_{1}(\mathbb{Q}) \mid\left(H_{i}^{1}((u: v))\right)^{b} \leq B\right\}=+\infty
$$

et donc aussi

$$
N_{i}\left(X(\mathbb{Q}), \mathcal{L}_{a, b}, B\right)=\#\left\{P \in X(\mathbb{Q}) \mid H_{a, b}^{i}(P) \leq B\right\}=+\infty .
$$

Cela permet de voir facilement que la série

$$
Z_{X}^{i}\left(\mathcal{L}_{a, b} ; s\right)=\sum_{P \in X(\mathbb{Q})}\left(H_{a, b}^{i}\right)^{-s}(P)
$$

ne converge pour aucune valeur de $s$ quand $b \leq 0$. Cette remarque explique pourquoi dans l'étude des fonctions zêta associées à tout le plan projectif éclaté en un point, on suppose en plus que $b>0$. Dans ce cas, par un calcul facile (voir les relations $(22)$ et $(23)$ ci-dessous $)$ on a $Z_{X}^{i}\left(\mathcal{L}_{a, b} ; s\right)=Z_{U}^{i}\left(\mathcal{L}_{a, b} ; s\right)+Z_{1, i}(b s)$. Cette dernière relation permet de voir qu'il suffit de donner les résultats concernant les fonctions zêta associées à l'ouvert $U=X \backslash E$.

Ceci étant, pour tout $i=\infty, 2$ on a :

THÉORÈme 2.5. - La fonction zêta de hauteur

$$
Z_{U}^{i}\left(\mathcal{L}_{a, b} ; s\right)=\sum_{P \in U(\mathbb{Q})}\left(H_{a, b}^{i}\right)^{-s}(P)
$$

TOME $133-2005-\mathrm{N}^{\mathrm{O}} 2$ 
converge absolument dans le demi plan $\{\operatorname{Re}(s)>\alpha\}$ et $\alpha$ est son abscisse de convergence absolue. De plus, elle possède un prolongement méromorphe à tout le plan complexe avec des pôles contenus dans l'ensemble $\mathcal{S}_{i}(U)$ où

$$
\begin{aligned}
\mathcal{S}_{\infty}(U) & =\left\{\frac{2}{a}, \frac{3-k}{a+b}, \frac{\beta}{a}, \frac{1+\beta-k}{a+b} \mid k \in \mathbb{N}, \beta \in \mathcal{Z}(\zeta)\right\}, \\
\mathcal{S}_{2}(U) & =\left\{\frac{2}{a}, \frac{3}{a+b}, \frac{2}{a+b}\right\} \cup\left\{\frac{1-2 k}{a}, \frac{\beta}{a}, \frac{1+\beta}{a+b} \mid k \in \mathbb{N}, \beta \in \mathcal{Z}(\zeta)\right\}
\end{aligned}
$$

et où $\mathcal{Z}(\zeta)$ est l'ensemble des zéros de la fonction zêta de Riemann $\zeta$.

ThÉorème 2.6. - 1) Pour tout $s \in \mathbb{C}$ vérifiant $\operatorname{Re}(s)>\alpha$, on a

$$
\begin{aligned}
& Z_{U}^{\infty}\left(\mathcal{L}_{a, b} ; s\right)= \frac{8 a s \zeta(a s-1) \zeta((a+b) s-2)}{(a s-1) \zeta(a s) \zeta((a+b) s-1)}+R_{U}^{\infty}\left(\mathcal{L}_{a, b} ; s\right) \\
& Z_{U}^{2}\left(\mathcal{L}_{a, b} ; s\right)= \frac{2 \sqrt{\pi} \Gamma\left(\frac{1}{2}(a s-1)\right) \zeta(a s-1) G((a+b) s-1)}{\Gamma\left(\frac{1}{2} a s\right) \zeta(a s) \zeta((a+b) s-1)} \\
&+\frac{4 G(a s)}{\zeta(a s)}+R_{U}^{2}\left(\mathcal{L}_{a, b} ; s\right)
\end{aligned}
$$

où $\Gamma$ est la fonction Gamma d'Euler, $G$ la fonction définie au $\S 1.2 .3$ (voir aussi le lemme 3.7) et où pour $i \in\{\infty, 2\}, s \mapsto R_{U}^{i}\left(\mathcal{L}_{a, b} ; s\right)$ est une fonction holomorphe dans le demi-plan $\left\{\operatorname{Re}(s)>\alpha^{\prime}\right\}$ et y vérifie

$$
R_{U}^{i}\left(\mathcal{L}_{a, b} ; s\right) \ll_{\operatorname{Re}(s)} 1+|\operatorname{Im}(s)| .
$$

2) En particulier, pour $i \in\{\infty, 2\}$, $\alpha$ est le seul pôle de $s \mapsto Z_{U}^{i}\left(\mathcal{L}_{a, b} ; s\right)$ dans $\left\{\operatorname{Re}(s)>\alpha^{\prime}\right\}$. De plus, pour tout $\varepsilon>0$, on a

$$
Z_{U}^{i}\left(\mathcal{L}_{a, b} ; s\right) \ll_{\sigma, \varepsilon} 1+|\tau|^{A(\alpha-\sigma)+\varepsilon}
$$

uniformément en $s=\sigma+i \tau \in \mathbb{C}$ vérifiant $\sigma>\alpha^{\prime}$ et $|\tau| \geq 1$, où $A=1 /\left(\alpha-\alpha^{\prime}\right)$ si $a \neq 2 b$ et $A=5 /\left(3\left(\alpha-\alpha^{\prime}\right)\right)=5 b$ si $a=2 b$.

3) En outre,

(a) si $a>2 b$, alors $\alpha=3 /(a+b)$ et c'est un pôle simple de résidu

$$
\operatorname{Res}_{s=\alpha} Z_{U}^{i}\left(\mathcal{L}_{a, b} ; s\right)= \begin{cases}\frac{144 a \zeta((2 a-b) /(a+b))}{\pi^{2}(2 a-b)(a+b) \zeta(3 a /(a+b))} & \text { si } i=\infty, \\ \frac{6 \Gamma((2 a-b) / 2(a+b)) \zeta((2 a-b) /(a+b))}{\sqrt{\pi}(a+b) \Gamma(3 a / 2(a+b)) \zeta(3 a /(a+b))} & \text { si } i=2 ;\end{cases}
$$

(b) si $a<2 b$, alors $\alpha=2 / a$ et c'est un pôle simple de résidu

$$
\operatorname{Res}_{s=\alpha} Z_{U}^{i}\left(\mathcal{L}_{a, b} ; s\right)= \begin{cases}\frac{96 \zeta(2 b / a)}{a \pi^{2} \zeta((a+2 b) / a)} & \text { si } i=\infty, \\ \frac{12 G((a+2 b) / a)}{a \pi \zeta((a+2 b) / a)}+\frac{12}{a \pi} & \text { si } i=2 .\end{cases}
$$

BULlETin DE LA SOCiÉtÉ MATHÉMATIQUe DE FRANCE 
(c) Si $a=2 b$, alors $\alpha=2 / a=1 / b$ et c'est un pôle double. La partie polaire de $Z_{U}^{i}\left(\mathcal{L}_{a, b} ; s\right)$, quand s tend vers $\alpha$, est donnée par

$$
\begin{aligned}
Z_{U}^{\infty}\left(\mathcal{L}_{a, b} ; s\right)= & \frac{96}{b^{2} \pi^{4}} \frac{1}{(s-\alpha)^{2}}+\frac{96}{b \pi^{6}}\left[(5 \gamma-1) \pi^{2}-30 \zeta^{\prime}(2)\right] \frac{1}{s-\alpha}+\mathcal{O}(1), \\
Z_{U}^{2}\left(\mathcal{L}_{a, b} ; s\right)= & \frac{6}{\pi^{2} b^{2}} \frac{1}{(s-\alpha)^{2}} \\
& \quad+\frac{6}{\pi^{4} b} \frac{6 g \pi+2 \gamma \pi^{2}-2 \log (2) \pi^{2}-30 \zeta^{\prime}(2)+\pi^{3}}{s-\alpha}+\mathcal{O}(1)
\end{aligned}
$$

où $\gamma$ est la constante d'Euler et où $g:=\lim _{s \rightarrow 0}(G(2+s)-\pi /(2 s))$ (cette limite existe d'après le lemme 3.7).

COROllaire 2.7. - Soient $a$ et $b$ deux entiers relatifs vérifiant $a+b>0$ et $a>0$. Alors pour $i \in\{\infty, 2\}$ et pour tout $\varepsilon>0$ on a, lorsque $B \rightarrow+\infty$,

$$
\begin{array}{r}
N_{i}\left(U(\mathbb{Q}), \mathcal{L}_{a, b} ; B\right)=B^{f_{i}(U, a, b)}\left[\theta_{i}(U, a, b)(\log B)^{g_{i}(U, a, b)}+\theta_{i}^{\prime}(U, a, b)\right] \\
+\mathcal{O}_{\varepsilon}\left(B^{f_{i}(U, a, b)-h_{i}(U, a, b)+\varepsilon}\right) .
\end{array}
$$

Les différentes constantes sont données par les tableaux de la page suivante où $g$ est la constante définie dans le théorème 2.6 .

\section{Préliminaires}

Nous regroupons dans cette partie quelques résultats qui nous seront utiles dans la suite.

Proposition 3.1 (théorème taubérien de Landau). - Soient $\left(a_{n}\right)_{n \geq 1}$ une suite d'éléments de $\mathbb{C}$ et $1 \leq \lambda_{1}<\cdots<\lambda_{n}<\cdots$ une suite strictement croissante de réels. On considère la série de Dirichlet

$$
s \longmapsto Z(s)=\sum_{n=1}^{+\infty} a_{n} \lambda_{n}{ }^{-s} \quad(s \in \mathbb{C}) \text {. }
$$

On suppose que:

1) $Z(s)$ converge absolument dans un demi-plan de la forme $\{\operatorname{Re}(s)>\alpha\}$. On notera $\sigma_{a}$ son abscisse de convergence absolue.

2) Il existe $\delta>0$ tel que $s \mapsto Z(s)$ possède un prolongement méromorphe au demi-plan $\left\{\operatorname{Re}(s)>\sigma_{a}-\delta\right\}$ avec un nombre fini de pôles qui sont tous réels. On note $\left(\sigma_{k}\right)_{0 \leq k \leq r}$ la famille finie et strictement décroissante des pôles de $s \mapsto s^{-1} Z(s)$ dans ce demi-plan.

3) Il existe $A>0$ telle que

$$
\forall \varepsilon>0, \quad Z(s) \ll_{\sigma, \varepsilon} 1+|\tau|^{A\left(\sigma_{0}-\sigma\right)+\varepsilon} \quad\left(\sigma>\sigma_{a}-\delta \text { et }|\tau| \geq 1\right) .
$$

TOME $133-2005-\mathrm{N}^{\mathrm{O}} 2$ 


\begin{tabular}{|c|c|c|c|c|c|}
\hline & $a<\frac{b}{2}$ & $\frac{b}{2} \leq a<2 b$ & $a>2 b, b \geq 0$ & $a>2 b, b<0$ & $a=2 b$ \\
\hline$h_{i}$ & $\frac{1}{2 a}$ & $\frac{2 b-a}{2 a(a+b)}$ & $\frac{a-2 b}{2 a(a+b)}$ & $\frac{1}{2(a+b)}$ & $\frac{1}{5 a}$ \\
\hline
\end{tabular}

\begin{tabular}{|c|c|c|c|c|}
\hline & $f_{\infty}$ & $g_{\infty}$ & $\theta_{\infty}$ & $\theta_{\infty}^{\prime}$ \\
\hline$a<2 b$ & $\frac{2}{a}$ & 0 & 0 & $\frac{48 \zeta(2 b / a)}{\pi^{2} \zeta((a+2 b) / a)}$ \\
\hline$a>2 b$ & $\frac{3}{a+b}$ & 0 & 0 & $\frac{48 a \zeta((2 a-b) /(a+b))}{(2 a-b) \pi^{2} \zeta(3 a /(a+b))}$ \\
\hline$a=2 b$ & $\frac{2}{a}=\frac{1}{b}$ & 1 & $\frac{96}{b \pi^{4}}$ & $\frac{96}{\pi^{6}}\left((5 \gamma-2) \pi^{2}-30 \zeta^{\prime}(2)\right)$ \\
\hline
\end{tabular}

\begin{tabular}{|c|c|c|c|c|}
\hline & $f_{2}$ & $g_{2}$ & $\theta_{2}$ & $\theta_{2}^{\prime}$ \\
\hline$a<2 b$ & $\frac{2}{a}$ & 0 & 0 & $\frac{6 G((a+2 b) / a)}{\pi \zeta((a+2 b) / a)}+\frac{6}{\pi}$ \\
\hline$a>2 b$ & $\frac{3}{a+b}$ & 0 & 0 & $\frac{2 \Gamma((2 a-b) / 2(a+b)) \zeta((2 a-b) /(a+b))}{\sqrt{\pi} \Gamma(3 a / 2(a+b)) \zeta(3 a /(a+b))}$ \\
\hline$a=2 b$ & $\frac{2}{a}=\frac{1}{b}$ & 1 & $\frac{6}{b \pi^{2}}$ & $\frac{6}{\pi^{4}}\left(\pi^{3}+(2 \gamma-2 \log 2-1) \pi^{2}+6 g \pi-30 \zeta^{\prime}(2)\right)$ \\
\hline
\end{tabular}

TABLE 1. Constantes du corollaire 2.7

Pour tout $k=0, \ldots, r$, on définit $Q_{k} \in \mathbb{R}[X]$ par la relation

$$
Q_{k}(x)=\mathrm{e}^{-\sigma_{k} x} \operatorname{Res}_{s=\sigma_{k}}\left(\frac{Z(s)}{s} \mathrm{e}^{s x}\right) .
$$

On pose aussi $\mu=\sup (1 / \delta, A)>0$ et on désigne par $\mathbf{E}(x)$ la partie entière de $x$. Alors :

1) $Q_{k}$ est un polynôme de degré égal à l'ordre de $\sigma_{k}$ en tant que pôle de $Z(s) / s$ moins 1 .

2) Pour tout $\varepsilon>0$ et $x \rightarrow+\infty$, on a

$$
\sum_{\lambda_{n} \leq x} a_{n}=\sum_{k=0}^{r} x^{\sigma_{k}} Q_{k}(\log (x))+\mathcal{O}_{\varepsilon}\left(x^{\sigma_{0}-\mathbf{E}(\mu \delta) /(\mathbf{E}(\mu \delta)+1) \mu+\varepsilon}\right) .
$$

BULLETIN DE LA SOCIÉTÉ MATHÉMATIQUE DE FRANCE 
3) Si en outre $s \mapsto Z(s)$ possède un prolongement méromorphe à tout le plan complexe $\mathbb{C}$ avec des pôles formant une suite (finie ou infinie) décroissante de réels et si ce prolongement vérifie la relation (5) uniformément en $s=\sigma+i \tau \in \mathbb{C}$ vérifiant $|\tau| \geq 1$, alors pour tout $\varepsilon>0$, on a

$$
\sum_{\lambda_{n} \leq x} a_{n}=\sum_{k=0}^{m} x^{\sigma_{k}} Q_{k}(\log (x))+\mathcal{O}_{\varepsilon}\left(x^{\sigma_{0}-1 / A+\varepsilon}\right) \quad(x \rightarrow+\infty)
$$

où $\left(\sigma_{k}\right)_{k \geq 0}$ est la suite décroissante des pôles de $s^{-1} Z(s)$, l'entier $m$ est défini par la relation $\sigma_{m+1}<\sigma_{0}-A^{-1} \leq \sigma_{m}$ et les polynômes $Q_{k}$ par la relation (6).

La preuve est une simple adaptation de celle de Landau [10] (voir par exemple $[11$, Appendix B]).

Lemme 3.2. - Soient $P \in \mathbb{R}\left[X_{1}, \ldots, X_{n}\right]$ un polynôme elliptique de degré $d$ et $P_{d}$ sa partie homogème de plus haut degré. Il existe alors $R>0$ tel que $P(x) \asymp P_{d}(x) \asymp\|x\|^{d}$ uniformément en $x \in \mathbb{R}_{+}^{n}$ vérifiant $\|x\| \geq R$.

Preuve du lemme 3.2. - Par compacité, il est bien évident que

$$
c_{1}=\inf _{\substack{x \in \mathbb{R}_{+}^{n} \\\|x\|=1}} P_{d}(x)>0 \quad \text { et } \quad c_{2}=\sup _{\substack{x \in \mathbb{R}_{+}^{n} \\\|x\|=1}} P_{d}(x)>0 .
$$

L'homogénéité de $P_{d}$ entraîne que l'on a $c_{1}\|x\|^{d} \leq P_{d}(x) \leq c_{2}\|x\|^{d}$ pour tout $x \in \mathbb{R}_{+}^{n}$. Le lemme découle alors du fait que $P(x)=P_{d}(x)+\mathcal{O}\left(\|x\|^{d-1}\right)$ lorsque $\|x\|$ tend vers l'infini.

Lemme 3.3. - Soit $A$ un sous-ensemble de $\mathbb{R}^{n}(n \geq 2)$ vérifiant $\lambda x \in A$ pour tout $x \in A$ et tout $\lambda \in \mathbb{Q}_{+}^{*}$. Soient $G: A \cap \mathbb{Z}^{n} \mapsto \mathbb{R}_{+}^{*}$ une fonction homogène de degré $g$ et $Q: A \cap \mathbb{Z}^{n} \mapsto \mathbb{C}$ une fonction homogène de degré $q$. On définit :

$$
\begin{aligned}
& Z_{A}(G, Q ; s)=\sum_{Z_{A, \operatorname{proj}}(G, Q ; s)}=\sum_{\substack{\left(m_{1}, \ldots, m_{n}\right) \in A \cap \mathbb{Z}^{n} \\
\operatorname{lm}\left(m_{1}, \ldots, m_{n}\right) \in A \cap \mathbb{Z}^{n}}} \frac{Q\left(m_{1}, \ldots, m_{n}\right)}{G^{s}\left(m_{1}, \ldots, m_{n}\right)}, \\
& \frac{Q\left(m_{1}, \ldots, m_{n}\right)}{G^{s}\left(m_{1}, \ldots, m_{n}\right)} .
\end{aligned}
$$

On suppose qu'il existe $\alpha>0$ tel que $s \mapsto Z_{A}(G, Q ; s)$ converge absolument dans le demi-plan $\{\operatorname{Re}(s)>\alpha\}$. Alors $s \mapsto Z_{A \text {,proj }}(G, Q ; s)$ converge aussi absolument dans le même demi-plan. De plus, pour tout $s \in \mathbb{C}$ vérifiant $\operatorname{Re}(s)>\alpha$,

$$
Z_{A, \operatorname{proj}}(G, Q ; s)=\zeta(g s-q)^{-1} Z_{A}(G, Q ; s) .
$$

TOME $133-2005-\mathrm{N}^{\mathrm{O}} 2$ 
Preuve du lemme 3.3. - Soit $s \in \mathbb{C}$ un complexe vérifiant $\operatorname{Re}(s)>\alpha$; il est clair alors que $Z_{A, \text { proj }}(G, Q ; s)$ converge absolument et qu'on a

$$
\begin{aligned}
& Z_{A, \operatorname{proj}}(G, Q ; s)=\sum_{\substack{\boldsymbol{m}=\left(m_{1}, \ldots, m_{n}\right) \in A \cap \mathbb{Z}^{n} \\
\operatorname{pgcd}\left(m_{1}, \ldots, m_{n}\right)=1}} \frac{Q(\boldsymbol{m})}{G^{s}(\boldsymbol{m})} \\
& =\sum_{\left(m_{1}, \ldots, m_{n}\right) \in A \cap \mathbb{Z}^{n}} \frac{Q(\boldsymbol{m})\left(\sum_{d \mid \operatorname{pgcd}\left(m_{1}, \ldots, m_{n}\right)} \mu(d)\right)}{G^{s}(\boldsymbol{m})} \\
& =\sum_{d=1}^{+\infty} \sum_{\substack{\left(m_{1}, \ldots, m_{n}\right) \in A \cap \mathbb{Z}^{n} \\
d\left|m_{1}, \ldots, d\right| m_{n}}} \frac{Q\left(m_{1}, \ldots, m_{n}\right) \mu(d)}{G^{s}\left(m_{1}, \ldots, m_{n}\right)} \\
& =\sum_{d=1}^{+\infty} \sum_{\left(m_{1}, \ldots, m_{n}\right) \in A \cap \mathbb{Z}^{n}} \frac{Q\left(d m_{1}, \ldots, d m_{n}\right) \mu(d)}{G^{s}\left(d m_{1}, \ldots, d m_{n}\right)} \\
& =\sum_{d=1}^{+\infty} \frac{\mu(d)}{d^{g s-q}} \sum_{\left(m_{1}, \ldots, m_{n}\right) \in A \cap \mathbb{Z}^{n}} \frac{Q\left(m_{1}, \ldots, m_{n}\right)}{G^{s}\left(m_{1}, \ldots, m_{n}\right)}=\frac{1}{\zeta(g s-q)} Z_{A}(G, Q ; s) .
\end{aligned}
$$

Lemme 3.4. - Soient $\lambda, \mu \in \mathbb{C}$ vérifiant $\operatorname{Re}(\lambda+\mu)>2$ et $\operatorname{Re}(\lambda)>1$. Alors la série

$$
\sum_{\substack{(k, \ell) \in\left(\mathbb{N}^{*}\right)^{2} \\ k \geq \ell}} \frac{1}{k^{\lambda}(n(k, \ell))^{\mu}}:=\sum_{\substack{(k, \ell) \in\left(\mathbb{N}^{*}\right)^{2} \\ k \geq \ell}} \frac{1}{k^{\lambda}(k / \operatorname{pgcd}(k, \ell))^{\mu}}
$$

converge absolument et vaut $\zeta(\lambda) \zeta(\lambda+\mu-1) / \zeta(\lambda+\mu)$.

Preuve du lemme 3.4. - Soient $\lambda, \mu \in \mathbb{R}$ vérifiant $\lambda+\mu>2$ et $\lambda>1$, dans $\overline{\mathbb{R}}_{+}$. On a alors :

$$
\begin{gathered}
\sum_{\substack{(k, \ell) \in\left(\mathbb{N}^{*}\right)^{2} \\
k \geq \ell}} \frac{1}{k^{\lambda}(k / \operatorname{pgcd}(k, \ell))^{\mu}}=\sum_{d=1}^{+\infty} \sum_{\substack{k \geq \ell \\
\operatorname{pgcd}(k, \ell)=d}} \frac{1}{k^{\lambda}(k / d)^{\mu}} \\
=\sum_{d=1}^{+\infty} \sum_{\substack{k \geq \ell \\
\operatorname{pgcd}(k, \ell)=1}} \frac{1}{(d k)^{\lambda} k^{\mu}}=\zeta(\lambda) \sum_{\substack{k \geq \ell \\
\operatorname{pgcd}(k, \ell)=1}} \frac{1}{k^{\lambda+\mu}} \\
=\frac{\zeta(\lambda)}{\zeta(\lambda+\mu)} \sum_{k \geq \ell} \frac{1}{k^{\lambda+\mu}} \quad(\text { d'après le lemme 3.3) } \\
=\frac{\zeta(\lambda)}{\zeta(\lambda+\mu)} \sum_{k \geq 1} \frac{k}{k^{\lambda+\mu}}=\frac{\zeta(\lambda) \zeta(\lambda+\mu-1)}{\zeta(\lambda+\mu)}<+\infty .
\end{gathered}
$$

Cela montre en particulier que la série converge absolument dans le domaine

$$
\left\{(\lambda, \mu) \in \mathbb{C}^{2} \mid \operatorname{Re}(\lambda+\mu)>2, \operatorname{Re}(\lambda)>1\right\}
$$

et qu'on a la même identité dans ce domaine par prolongement analytique.

BULlETin DE LA SOCiÉtÉ MATHÉMATIQUe DE FRANCE 
Lemme 3.5. - Soit $N \in \mathbb{N}$. Alors pour tout $x \in \mathbb{N}^{*}$ et tout $s \in \mathbb{C}$ vérifiant $\operatorname{Re}(s)>1$, on $a$

$$
\sum_{k>x} \frac{1}{k^{s}}=\frac{1}{(s-1) x^{s-1}}+\sum_{k=0}^{N} \frac{(-1)^{k} B_{k+1}\left(\begin{array}{l}
-s \\
k
\end{array}\right)}{(k+1) x^{s+k}}+R_{N}(x ; s)
$$

où

1) $B_{k}$ est le k-ième nombre de Bernoulli pour tout $k \in \mathbb{N}$,

2) $x \mapsto R_{N}(x ; s)$ est holomorphe dans le demi-plan $\{\operatorname{Re}(s)>-N\}$ et $y$ vérifie $R_{N}(x ; s) \ll_{N, \sigma}\left(1+|\tau|^{N+1} / x^{\sigma+N}\right)$ uniformément en $x \in \mathbb{N}^{*}$ et en $s=\sigma+i \tau$ tel que $\sigma>-N$.

Ce lemme se démontre facilement en appliquant la formule d'Euler-Mac Laurin (voir par exemple $\left[17\right.$, p. 6, th. 4]) à la fonction $f(t)=t^{-s}$.

Lemme 3.6. - Soit $\alpha \in \mathbb{R}_{+}^{*}$. On considère la série

$$
F(\alpha ; s)=\sum_{k=1}^{+\infty} \frac{1}{{\sqrt{\alpha^{2}+k^{2}}}^{s}} .
$$

Alors, $s \mapsto F(\alpha ; s)$ converge absolument dans le demi-plan $\{\operatorname{Re}(s)>1\}$ et $y$ vérifie

$$
F(\alpha ; s)=\frac{\sqrt{\pi} \Gamma\left(\frac{1}{2}(s-1)\right)}{2 \Gamma\left(\frac{1}{2} s\right) \alpha^{s-1}}-\frac{1}{2 \alpha^{s}}+R(\alpha ; s)
$$

où $s \mapsto R(\alpha ; s)$ est une fonction entière vérifiant, pour tout $N \in \mathbb{N}$,

$$
R(\alpha ; s) \ll_{\sigma, N} \frac{1+|\tau|^{N+1}}{\alpha^{\sigma+N}}
$$

uniformément en s tel que $\sigma>-N$ et en $\alpha \geq 1$.

Preuve du lemme 3.6. - On fixe $\alpha>0$ et $s \in \mathbb{C}$ tel que $\operatorname{Re}(s)>1$ et on pose $f(x)=\left(\alpha^{2}+x^{2}\right)^{-s / 2}$ pour tout $x \in \mathbb{R}$. Il est facile de voir que pour tout $k \in \mathbb{N}$, $x \in \mathbb{R}, \alpha \geq 1$ et $s \in \mathbb{C}$, on a

$$
\begin{array}{r}
f^{(2 k)}(0)=\frac{(2 k) !\left(\begin{array}{c}
-s / 2 \\
k
\end{array}\right)}{\alpha^{s+2 k}}, \quad f^{(2 k+1)}(0)=0 \quad \text { et } \\
f^{(k)}(x) \ll_{k} \frac{1+|s|^{k}}{\left(\alpha^{2}+x^{2}\right)^{(\operatorname{Re}(s)+k) / 2}} .
\end{array}
$$

En particulier, on a $\lim _{x \rightarrow+\infty} f^{(k)}(x)=0$ pour tout $k \in \mathbb{N}$ et $B_{k+1} f^{(k)}(0)=0$ pour tout $k \in \mathbb{N}^{*}$. Soit $N \in \mathbb{N}$; la formule d'Euler-Mac Laurin appliquée à 
l'ordre $N$ à la fonction $f$ implique alors que

$$
\begin{aligned}
R(\alpha ; s) & :=F(\alpha ; s)-\int_{0}^{+\infty} \frac{\mathrm{d} x}{\left(x^{2}+\alpha^{2}\right)^{s / 2}}+\frac{1}{2 \alpha^{s}} \\
& =\frac{(-1)^{N}}{(N+1) !} \int_{0}^{+\infty} B_{N+1}(x) f^{(N+1)}(x) \mathrm{d} x .
\end{aligned}
$$

D'autre part, la relation (7) implique que pour $\sigma=\operatorname{Re}(s)>-N$ et pour $\alpha \geq 1$ :

$$
\begin{aligned}
\int_{0}^{+\infty}\left|B_{N+1}(x) f^{(N+1)}(x)\right| \mathrm{d} x & \ll_{N} \frac{1+|s|^{N+1}}{\alpha^{\sigma+N}} \int_{0}^{+\infty} \frac{\mathrm{d} y}{\left(1+y^{2}\right)^{(\sigma+N+1) / 2}} \\
& \ll_{N, \sigma} \frac{1+|s|^{N+1}}{\alpha^{\sigma+N}} .
\end{aligned}
$$

On en déduit que $s \mapsto R(\alpha ; s)$ est holomorphe dans le demi-plan $\operatorname{Re}(s)>-N$ et y vérifie $R(\alpha ; s) \ll_{N, \sigma}\left(1+|\tau|^{N+1}\right) / \alpha^{\sigma+N}$ uniformément en $s=\sigma+i \tau$ tel que $\sigma>-N$ et en $\alpha \geq 1$. Comme il est clair que la fonction $s \mapsto R(\alpha ; s)$ ne dépend pas de $N$, donc en faisant tendre $N$ vers l'infini, on déduit que cette fonction est entière. Pour terminer la preuve de ce lemme, il suffit donc de vérifier que pour tout $s \in \mathbb{C}$ tel que $\operatorname{Re}(s)>1$, on a

$$
\int_{0}^{+\infty} \frac{\mathrm{d} x}{\left(x^{2}+\alpha^{2}\right)^{s / 2}}=\frac{\sqrt{\pi} \Gamma\left(\frac{1}{2}(s-1)\right)}{2 \alpha^{s-1} \Gamma\left(\frac{1}{2} s\right)} .
$$

On l'obtient en effectuant le changement de variables $t=\alpha^{2} /\left(\alpha^{2}+x^{2}\right)$ et en utilisant l'identité des compléments $B(\lambda, \mu)=\int_{0}^{1} t^{\lambda-1}(1-t)^{\mu-1} \mathrm{~d} t=\frac{\Gamma(\lambda) \Gamma(\mu)}{\Gamma(\lambda+\mu)}$, valable pour $\operatorname{Re}(\lambda)>0$ et $\operatorname{Re}(\mu)>0$.

Lemme 3.7. - On considère la série de Dirichlet

$$
G(s)=\sum_{k=1}^{+\infty} \sum_{\ell=1}^{+\infty} \frac{1}{{\sqrt{\left(k^{2}+\ell^{2}\right)}}^{s}}
$$

Alors :

1) $s \mapsto G(s)$ converge absolument dans le demi-plan $\{\operatorname{Re}(s)>2\}$ et y vérifie

$$
G(s)=\frac{\sqrt{\pi} \Gamma\left(\frac{1}{2}(s-1)\right)}{2 \Gamma(1 / 2 s)} \zeta(s-1)-\frac{1}{2} \zeta(s)+L(s)
$$

où $s \mapsto L(s)$ est une fonction entière vérifiant $L(s) \ll_{\sigma, N} 1+|\tau|^{N+1}$ pour tout $N \in \mathbb{N}$, uniformément en $s=\sigma+i \tau$ tel que $\sigma>1-N$.

2) En particulier, $s \mapsto G(s)$ possède un prolongement méromorphe à tout le plan complexe avec comme seuls pôles 2 et 1 . Ces pôles sont simples de résidus donnés par

$$
\operatorname{Res}_{s=2} G=\frac{\pi}{2} \quad \text { et } \quad \operatorname{Res}_{s=1} G=-1 .
$$

En plus $\sigma_{a}=2$ est l'abscisse de convergence de $G$.

BULLETIN DE LA SOCIÉTÉ MATHÉMATIQUE DE FRANCE 
3) On a $G(\sigma+i \tau) \ll_{\sigma, \varepsilon} 1+|\tau|^{2-\sigma+\varepsilon}$ pour tout $\varepsilon>0(\sigma, \tau \in \mathbb{R}$ et $|\tau| \geq 1)$.

Remarque. - $G(s)$ est une série de Dirichlet classique qui est liée aux fonctions $L$ de Dirichlet par la formule suivante (voir [8, th. 306, p. 256]) :

$$
G(s)=\frac{1}{4} \sum_{n=1}^{+\infty} \frac{r(n)}{n^{s / 2}}-\zeta(s)=\zeta\left(\frac{s}{2}\right) L\left(\frac{s}{2}, \chi\right)-\zeta(s)
$$

où pour tout $n \in \mathbb{N}^{*}, r(n)=\#\left\{(k, \ell) \in \mathbb{Z}^{2} \mid n=k^{2}+\ell^{2}\right\}$ et où $\chi$ désigne le caractère non principal modulo 4. Évidemment, la relation (8) permet de retrouver le lemme 3.7. Néanmoins nous donnons, par souci de simplicité, une preuve courte et indépendante de ce lemme :

Preuve du lemme 3.7. - Avec les notations du lemme 3.6, on a pour $N \in \mathbb{N}$ et $\operatorname{Re}(s)>2$ :

$$
\begin{aligned}
G(s) & =\sum_{k=1}^{+\infty} \sum_{\ell=1}^{+\infty} \frac{1}{{\sqrt{k^{2}+\ell^{2}}}^{s}}=\sum_{k=1}^{+\infty} F(k ; s)=\sum_{k=1}^{+\infty} \frac{\sqrt{\pi} \Gamma\left(\frac{1}{2}(s-1)\right)}{2 \Gamma\left(\frac{1}{2} s\right) k^{s-1}}-\frac{1}{2 k^{s}}+R(k ; s) \\
& =\frac{\sqrt{\pi} \Gamma\left(\frac{1}{2}(s-1)\right)}{2 \Gamma\left(\frac{1}{2} s\right)} \zeta(s-1)-\frac{1}{2} \zeta(s)+L(s) \quad \text { où } \quad L(s)=\sum_{k=1}^{+\infty} R(k ; s) .
\end{aligned}
$$

Les propriétés de $s \mapsto R(\alpha ; s)$ établies au lemme 3.6 et celles classiques de la fonction zêta de Riemann, permettent alors de conclure.

Proposition 3.8. - On considère les fonctions zêta associées à la droite projective définies par la relation (2). Alors, pour $i=\infty, 2$, les séries

$$
\begin{gathered}
s \longmapsto Z_{1, i}(s)=\sum_{P=\left(x_{0}: x_{1}\right) \in \mathbb{P}_{1}(\mathbb{Q})} \frac{1}{H_{i}^{1}(P)^{s}}, \\
s \longmapsto Z_{1, i}^{*}(s)=\sum_{\substack{P=\left(x_{0}: x_{1}\right) \in \mathbb{P}_{1}(\mathbb{Q}) \\
x_{0} x_{1} \neq 0}} \frac{1}{H_{i}^{1}(P)^{s}}
\end{gathered}
$$

convergent absolument dans le demi-plan $\{\operatorname{Re}(s)>2\}$ et y vérifient

$$
Z_{1, \infty}(s)=Z_{1, \infty}^{*}(s)+2=\frac{4 \zeta(s-1)}{\zeta(s)}, \quad Z_{1,2}(s)=Z_{1,2}^{*}(s)+2=\frac{2 G(s)}{\zeta(s)}+2
$$

où $\zeta$ est la fonction Zêta de Riemann et $G(s)=\sum_{k=1}^{+\infty} \sum_{\ell=1}^{+\infty}\left(k^{2}+\ell^{2}\right)^{-s / 2}$.

Preuve de la proposition 3.8. - Cas $i=\infty$ : c'est un cas particulier de la proposition 2.1 que nous démontrerons dans $\S 4.1$.

Cas $i=2$ : le lemme 3.3 implique que pour $\operatorname{Re}(s)$ assez grande, on a

$$
Z_{1,2}(s)-2=Z_{1,2}^{*}(s)=2 \sum_{\substack{(k, \ell) \in \mathbb{N}^{* 2} \\ \operatorname{pgcd}(k, \ell)=1}} \frac{1}{{\sqrt{k^{2}+\ell^{2}}}^{s}}=\frac{2 G(s)}{\zeta(s)} .
$$


Lemme 3.9. - Pour tous $\mu, \delta \in \mathbb{C}$ tels que $\operatorname{Re}(\mu)>1$ et $\operatorname{Re}(\mu+\delta)>2$, la série

$$
\sum_{(x, y) \in \mathbb{N}^{* 2}} \frac{1}{\sqrt{n(x, y)^{2}+n(y, x)^{2}}} \delta \frac{1}{{\sqrt{x^{2}+y^{2}}}^{\mu}}
$$

converge absolument et vaut $\zeta(\mu) \zeta(\mu+\delta)^{-1} G(\mu+\delta)$ où $G$ est la fonction définie dans le lemme 3.7 .

Preuve du lemme 3.9. - Le lemme 3.3 entraîne qu'on a formellement :

$$
\begin{aligned}
& \sum_{(x, y) \in \mathbb{N}^{* 2}} \frac{1}{{\sqrt{n(x, y)^{2}+n(y, x)^{2}}}^{\delta}} \frac{1}{{\sqrt{x^{2}+y^{2}}}^{\mu}} \\
& =\sum_{d=1}^{+\infty} \sum_{\substack{(x, y) \in \mathbb{N}^{* 2} \\
\operatorname{pgcd}(x, y)=d}} \frac{1}{\sqrt{(x / d)^{2}+(y / d)^{2}}} \delta \frac{1}{{\sqrt{x^{2}+y^{2}}}^{\mu}} \\
& =\sum_{d=1}^{+\infty} \frac{1}{d^{\mu}} \sum_{\substack{(x, y) \in \mathbb{N}^{* 2} \\
\operatorname{pgcd}(x, y)=1}} \frac{1}{{\sqrt{x^{2}+y^{2}}}^{\delta}} \frac{1}{{\sqrt{x^{2}+y^{2}}}^{\mu}} \\
& =\zeta(\mu) \sum_{\substack{(x, y) \in \mathbb{N}^{* 2} \\
\operatorname{pgcd}(x, y)=1}} \frac{1}{{\sqrt{x^{2}+y^{2}}}^{\mu+\delta}}=\frac{\zeta(\mu) G(\mu+\delta)}{\zeta(\mu+\delta)} .
\end{aligned}
$$

En particulier, la série convergence absolument dans le domaine $\{\operatorname{Re}(\mu)>1$, $\operatorname{Re}(\mu+\delta)>2\}$.

\section{Preuve des résultats concernant $\mathbb{P}_{n}(\mathbb{Q})$}

\subsection{Preuve de la proposition 2.1 et du corollaire $\mathbf{2 . 2}$}

4.1.1. Étude de $Z_{n, \infty}^{*}(s)$. - Rappelons que $V_{n}(\mathbb{Q})=\left\{x_{0} \cdots x_{n} \neq 0\right\} \subset \mathbb{P}_{n}(\mathbb{Q})$. La définition de $H_{\infty}$, permet de voir que dans son domaine de convergence absolue, la série $Z_{n, \infty}^{*}(s)$ vérifie

$$
Z_{n, \infty}^{*}(s)=2^{n} \sum_{\substack{\left(m_{0}, \ldots, m_{n}\right) \in\left(\mathbb{N}^{*}\right)^{n+1} \\ \operatorname{pgcd}\left(m_{0}, \ldots, m_{n}\right)=1}} \frac{1}{\left(\max _{0 \leq i \leq n} m_{i}\right)^{s}} .
$$

Par conséquent, $s \mapsto Z_{n, \infty}^{*}(s)$ converge absolument et est holomorphe dans $\{\operatorname{Re}(s)>n+1\}$. De plus, en utilisant le lemme 3.3 , on obtient pour tout $s \in \mathbb{C}$ vérifiant $\operatorname{Re}(s)>n+1$ :

$$
Z_{n, \infty}^{*}(s)=\frac{2^{n}}{\zeta(s)} \sum_{m_{0}, \ldots, m_{n} \geq 1} \frac{1}{\left(\max _{0 \leq i \leq n}\left(m_{i}\right)\right)^{s}}=\frac{2^{n}}{\zeta(s)} \sum_{k=1}^{+\infty} \frac{c_{k}}{k^{s}}
$$

BULlETIN DE LA SOCIÉTÉ MATHÉMATIQUE DE FRANCE 
où pour tout $k \in \mathbb{N}^{*}$

$$
\begin{aligned}
c_{k} & =\#\left\{\left(m_{0}, \ldots, m_{n}\right) \in\left(\mathbb{N}^{*}\right)^{n+1} \mid \max _{0 \leq i \leq n} m_{i}=k\right\} \\
& =k^{n+1}-(k-1)^{n+1}=\sum_{r=0}^{n}\left(\begin{array}{c}
n+1 \\
r+1
\end{array}\right) k^{n-r}(-1)^{r} .
\end{aligned}
$$

Les relations (9), (10) et une simple interversion de sommations permettent de voir que pour tout $s \in \mathbb{C}$ vérifiant $\operatorname{Re}(s)>n+1$ :

$$
Z_{n, \infty}^{*}(s)=\frac{2^{n}}{\zeta(s)} \sum_{\ell=0}^{n}(-1)^{n-\ell}\left(\begin{array}{c}
n+1 \\
\ell
\end{array}\right) \zeta(s-\ell) .
$$

Les propriétés classiques de la fonction zêta de Riemann permettent alors de montrer la proposition 2.1 pour $Z_{n, \infty}^{*}(s)$. Il reste à vérifier que $n+1$ est effectivement l'abscisse de convergence de $Z_{n, \infty}^{*}(s)$ : la relation (11) montre que $n+1$ est un pôle simple de résidu $2^{n}(n+1) / \zeta(n+1)$ qui est strictement positif. Par suite $s=n+1$ est la seule singularité de la série de Dirichlet à coefficients positifs $Z_{n, \infty}^{*}(s)$ dans le demi-plan $\{\operatorname{Re}(s) \geq n+1\}$. Le théorème de Landau (voir [9] ou $[17$, cor. 6.1 , p. 113]), implique alors que $n+1$ est l'abscisse de convergence de $Z_{n, \infty}^{*}(s)$. La proposition 2.1 est donc montrée pour $Z_{n, \infty}^{*}(s)$.

4.1.2. Étude de $Z_{n, \infty}(s)$. — Posons pour tout $I \subset\{0, \ldots, n\}$,

$$
V_{n}^{I}=\left\{\left(x_{0}: \ldots: x_{n}\right) \in \mathbb{P}_{n}(\mathbb{Q}) \mid x_{i} \neq 0, \forall i \in I \text { et } x_{i}=0, \forall i \notin I\right\} .
$$

Il est clair que

$$
\mathbb{P}_{n}(\mathbb{Q})=\bigcup_{\varnothing \neq I \subset\{0, \ldots, n\}}^{\bullet} V_{n}^{I} \quad \text { (la réunion est disjointe). }
$$

On obtient par symétrie, vu l'étude de $Z_{n, \infty}^{*}$, que pour $\operatorname{Re}(s)>n+1$ :

$$
\begin{aligned}
\sum_{r=1}^{n+1}\left(\begin{array}{c}
n+1 \\
r
\end{array}\right) Z_{r-1, \infty}^{*}(s) & =\sum_{r=1}^{n+1} \sum_{\substack{I \subset\{0, \ldots, n\} \\
\# I=r}} \sum_{M \in V_{n}^{I}(\mathbb{Q})} \frac{1}{H_{\infty}(M)^{s}} \\
& =\sum_{\varnothing \neq I \subset\{0, \ldots, n\}} \sum_{M \in V_{n}^{I}(\mathbb{Q})} \frac{1}{H_{\infty}(M)^{s}}=Z_{n, \infty}(s)
\end{aligned}
$$

On en déduit que $s \mapsto Z_{n}(s)$ converge absolument dans $\{\operatorname{Re}(s)>n+1\}$ et que

$$
\forall s \in \mathbb{C}, \operatorname{Re}(s)>n+1, \quad Z_{n, \infty}(s)=\sum_{r=0}^{n}\left(\begin{array}{c}
n+1 \\
r+1
\end{array}\right) Z_{r, \infty}^{*}(s) .
$$


La relation (11) implique alors que pour tout $s \in \mathbb{C}$ vérifiant $\operatorname{Re}(s)>n+1$, on a

$$
Z_{n, \infty}(s)=\frac{1}{\zeta(s)} \sum_{\ell=0}^{n} a(\ell, n) \zeta(s-\ell)
$$

où $a(\ell, n)=\sum_{r=\ell}^{n}\left(\begin{array}{c}n+1 \\ r+1\end{array}\right)\left(\begin{array}{c}r+1 \\ \ell\end{array}\right) 2^{r}(-1)^{r-\ell}$ pour tout $\ell \in\{0, \ldots, n\}$. Les propriétés classiques de la fonction zêta de Riemann permettent alors de conclure, comme dans la précédente section, que $Z_{n, \infty}(s)$ vérifie les conclusions de la proposition 2.1. Par conséquent, la démonstration de cette proposition est terminée.

4.1.3. Preuve du corollaire 2.2. - Le corollaire 2.2 découle immédiatement de l'identification des coefficients des séries de Dirichlet figurant à gauche et à droite des relations (3) et (4) de la proposition 2.1. On peut aussi le démontrer directement sans passer par les séries de Dirichlet.

4.2. Preuve du théorème 2.3 et du corollaire 2.4. - Dans toute cette partie, $P \in \mathbb{R}\left[X_{0}, \ldots, X_{n}\right]$ désigne un polynôme elliptique de degré $d \geq 1$.

4.2.1. Étude de $Z_{\text {proj }}^{*}(P ; s)$. — Comme dans le début de la section 4.1.1, il est clair que l'on a

$$
Z_{\mathrm{proj}}^{*}(P ; s)=\sum_{M \in V_{n}(\mathbb{Q})} \frac{1}{H_{P}(M)^{s}}=2^{n} \sum_{\begin{array}{c}
m_{0}, \ldots, m_{n} \geq 1 \\
\operatorname{pgcd}\left(m_{0}, \ldots, m_{n}\right)=1
\end{array}} \frac{1}{P\left(m_{0}, \ldots, m_{n}\right)^{s / d}}
$$

dans tout domaine de convergence absolue d'au moins une des deux séries.

On a $\sum_{m_{0}, \ldots, m_{n} \geq 1} P\left(m_{0}, \ldots, m_{n}\right)^{-\sigma / d}<+\infty$ pour $\sigma>n+1$ d'après le lemme 3.2. On en déduit que $s \mapsto Z_{\text {proj }}^{*}(P ; s)$ converge absolument dans le demi-plan $\{\operatorname{Re}(s)>n+1\}$ et y vérifie

$$
Z_{\text {proj }}^{*}(P ; s)=2^{n} \sum_{\substack{m_{0}, \ldots, m_{n} \geq 1 \\ \operatorname{pgcd}\left(m_{0}, \ldots, m_{n}\right)=1}} \frac{1}{P\left(m_{0}, \ldots, m_{n}\right)^{s / d}} .
$$

D'autre part, on a $P(X)=P_{d}(X)+P_{d-1}(X)+\cdots+P_{0}(X)$ où $P_{j}$ est la partie homogène de $P$ de degré $j$ pour tout $j=0, \ldots, d$. Par conséquent si l'on pose $H(X)=\sum_{j=0}^{d-1} P_{j}(X)$, le degré de $H$ est inférieur ou égal à $d-1$ et $H(x) \ll\|x\|^{d-1}$ uniformément sur $\left[1,+\infty\left[^{n+1}\right.\right.$. Comme en plus, le lemme 3.2 implique $P_{d}(x) \asymp\|x\|^{d}$ uniformément sur $\left[1,+\infty\left[^{n+1}\right.\right.$ on obtient alors

$$
\frac{H(x)}{P_{d}(x)} \ll \frac{1}{\|x\|} \quad \text { uniformément sur }\left[1,+\infty\left[^{n+1} .\right.\right.
$$

BULlETIN DE LA SOCIÉTÉ MATHÉMATIQUE DE FRANCE 
Soit maintenant $N$ un entier positif fixé mais arbitraire. En utilisant un développement de Taylor, on obtient uniformément en $s \in \mathbb{C}$ et $x \in\left[1,+\infty\left[^{n+1}\right.\right.$ :

$$
\begin{aligned}
P(x)^{-s / d} & =\left[P_{d}(x)+H(x)\right]^{-s / d}=P_{d}(x)^{-s / d}\left[1+\frac{H(x)}{P_{d}(x)}\right]^{-s / d} \\
& =\sum_{k=0}^{N}\left(\begin{array}{c}
-s / d \\
k
\end{array}\right) \frac{H(x)^{k}}{P_{d}(x)^{s / d+k}}+\mathcal{O}\left(\frac{1+|s|^{N+1}}{\|x\|^{N+1+\sigma}}\right) .
\end{aligned}
$$

On en déduit que pour tout $s \in \mathbb{C}$ vérifiant $\sigma=\operatorname{Re}(s)>n+1$, on a

$$
Z_{\text {proj }}^{*}(P ; s)=2^{n} \sum_{k=0}^{N}\left(\begin{array}{c}
-s / d \\
k
\end{array}\right) \sum_{\substack{m_{0}, \ldots, m_{n} \geq 1 \\
\operatorname{pgcd}\left(m_{0}, \ldots, m_{n}\right)=1}} \frac{H(m)^{k}}{P_{d}(m)^{s / d+k}}+\mathcal{R}_{N}(P ; s)
$$

où $s \mapsto \mathcal{R}_{N}(P ; s)$ est holomorphe dans le demi-plan $\{\operatorname{Re}(s)>n-N\}$ et y vérifie $\mathcal{R}_{N}(P ; s) \ll_{\operatorname{Re}(s)} 1+|s|^{N+1}$. Comme en plus

$$
\begin{aligned}
H(X)^{k} & =\left(P_{0}(X)+\cdots+P_{d-1}(X)\right)^{k} \\
& =\sum_{\substack{\alpha=\left(\alpha_{0}, \ldots, \alpha_{d-1}\right) \in \mathbb{N}^{d} \\
|\alpha|=\alpha_{0}+\cdots+\alpha_{d-1}=k}} \frac{k !}{\alpha_{0} ! \ldots \alpha_{d-1} !} P_{0}(X)^{\alpha_{0}} \cdots P_{d-1}(X)^{\alpha_{d-1}},
\end{aligned}
$$

alors pour tout $s \in \mathbb{C}$ vérifiant $\operatorname{Re}(s)>n+1$ :

$$
\begin{aligned}
Z_{\text {proj }}^{*}(P ; s) & =2^{n} \sum_{k=0}^{N}\left(\begin{array}{c}
-s / d \\
k
\end{array}\right) \sum_{\substack{\alpha \in \mathbb{N}^{d} \\
|\alpha|=k \operatorname{pgcd}\left(m_{0}, \ldots, m_{n}\right)=1}} \frac{k !}{\alpha !} \sum_{\substack{m_{0}, \ldots, m_{n} \geq 1 \\
P_{d}(m)^{s / d+k}}} \frac{\prod_{j=0}^{d-1} P_{j}(m)^{\alpha_{j}}}{P_{N}}+\mathcal{R}_{N}(P ; s) \\
& =2^{n} \sum_{\substack{\alpha \in \mathbb{N}^{d} \\
|\alpha| \leq N}}\left(\begin{array}{c}
-s / d \\
|\alpha|
\end{array}\right) \frac{|\alpha| !}{\alpha !} \sum_{\substack{m_{0}, \ldots, m_{n} \geq 1 \\
\operatorname{pgcd}\left(m_{0}, \ldots, m_{n}\right)=1}} \frac{\prod_{j=0}^{d-1} P_{j}(m)^{\alpha_{j}}}{P_{d}(m)^{s / d+|\alpha|}}+\mathcal{R}_{N}(P ; s) .
\end{aligned}
$$

Posons $T_{\alpha}(X)=\prod_{j=0}^{d-1} P_{j}(X)^{\alpha_{j}}$ pour tout $\alpha=\left(\alpha_{0}, \ldots, \alpha_{d-1}\right) \in \mathbb{N}^{d}$. Alors $T_{\alpha}$ est un polynôme homogène en $X=\left(X_{0}, \ldots, X_{n}\right)$ de degré $\sum_{j=0}^{d-1} j \alpha_{j}$. En utilisant le lemme 3.3, on obtient pour $\alpha \in \mathbb{N}^{d}$ et pour $\operatorname{Re}(s)>n+1$ :

$$
\begin{aligned}
& \sum_{\substack{m_{0}, \ldots, m_{n} \geq 1 \\
\operatorname{pgcd}\left(m_{0}, \ldots, m_{n}\right)=1}} \frac{\prod_{j=0}^{d-1} P_{j}(m)^{\alpha_{j}}}{P_{d}(m)^{s / d+|\alpha|}}=\sum_{\substack{m_{0}, \ldots, m_{n} \geq 1 \\
\operatorname{pgcd}\left(m_{0}, \ldots, m_{n}\right)=1}} \frac{T_{\alpha}(m)}{P_{d}(m)^{s / d+|\alpha|}} \\
& =\frac{1}{\zeta\left(s+d|\alpha|-\sum_{j=0}^{d-1} j \alpha_{j}\right)} \sum_{m_{0}, \ldots, m_{n} \geq 1} \frac{T_{\alpha}(m)}{P_{d}(m)^{s / d+|\alpha|}} .
\end{aligned}
$$

TOME $133-2005-\mathrm{N}^{\mathrm{O}} 2$ 
Donc pour tout $N \in \mathbb{N}$ et pour $\operatorname{Re}(s)>n+1$, on a

$$
Z_{\text {proj }}^{*}(P ; s)=2^{n} \sum_{\substack{\alpha \in \mathbb{N}^{d} \\
|\alpha| \leq N}}\left(\begin{array}{c}
-s / d \\
|\alpha|
\end{array}\right) \frac{|\alpha| !}{\alpha !} \frac{Z\left(P_{d}, T_{\alpha} ; s / d+|\alpha|\right)}{\zeta\left(s+d|\alpha|-\sum_{j=0}^{d-1} j \alpha_{j}\right)}+\mathcal{R}_{N}(P ; s)
$$

où $Z\left(P_{d}, T_{\alpha} ; s\right)=\sum_{m \in\left(\mathbb{N}^{*}\right)^{n+1}} T_{\alpha}(m) / P_{d}^{s}(m)$ et où $s \mapsto \mathcal{R}_{N}(P ; s)$ est holomorphe dans $\{\operatorname{Re}(s)>n-N\}$ et y vérifie $\mathcal{R}_{N}(P ; s) \ll_{\operatorname{Re}(s)} 1+|s|^{N+1}$.

D'autre part, il découle de la théorie générale des séries de Dirichlet associées à des polynômes de plusieurs variables (voir [12, Satz II, p. 397-398] pour le cas elliptique et [6] pour le cas général), que pour tout $\alpha=\left(\alpha_{0}, \ldots, \alpha_{d-1}\right) \in \mathbb{N}^{d}$, $s \mapsto Z\left(P_{d}, T_{\alpha} ; s / d+|\alpha|\right)$ converge absolument dans le demi-plan

$$
\left\{\operatorname{Re}(s)>n+1-\sum_{j=0}^{d-1}(d-j) \alpha_{j}\right\}
$$

et possède un prolongement méromorphe à tout le plan complexe $\mathbb{C}$ avec des pôles simples contenus dans l'ensemble

$$
S^{\prime}(\alpha)=\left\{n+1-\sum_{j=0}^{d-1}(d-j) \alpha_{j}-\ell \mid \ell \in \mathbb{N}\right\} .
$$

En outre, pour tout $N \in \mathbb{N}$, tout $\varepsilon>0$ et tout $s \in \mathbb{C}$ vérifiant $\sigma>n+1-N$ et $|\tau| \geq 1$, on a

$$
Z\left(P_{d}, T_{\alpha} ; \frac{s}{d}+|\alpha|\right) \ll_{N, \varepsilon} 1+|\tau|^{n+1-\sigma+\sum_{j=0}^{d-1} j \alpha_{j}-d|\alpha|+\varepsilon} .
$$

En utilisant les relations (17) et (18) et en faisant tendre $N$ vers l'infini, on déduit que $s \mapsto Z_{\text {proj }}^{*}(P ; s)$ possède un prolongement méromorphe à tout le plan complexe avec des pôles contenus dans l'ensemble

$$
S=\{n+1-\ell \mid \ell \in \mathbb{N}\} \cup\{\beta-j \mid \zeta(\beta)=0 \text { et } j \in \mathbb{N}\} .
$$

De plus, il est clair que :

1) $\{1,2, \ldots, n+1\}$ sont les seuls candidats-pôles dans le demi-plan fermé $\{\operatorname{Re}(s) \geq 1\}$ et qu'ils sont au plus simples.

2) $s=n+1$ est l'abscisse de convergence de $Z_{\text {proj }}^{*}(P ; s)$ et que c'est un pôle simple de résidu

$$
\begin{aligned}
\underset{s=n+1}{\operatorname{Res}} Z_{\text {proj }}^{*}(P ; s) & =\frac{2^{n}}{\zeta(n+1)} \operatorname{Res}_{s=n+1} Z\left(P_{d}, 1 ; \frac{s}{d}\right) \\
& =\frac{2^{n}}{\zeta(n+1)} \int_{S^{n} \cap \mathbb{R}_{+}^{n+1}} P_{d}(u)^{-(n+1) / d} \mathrm{~d} \sigma(u) .
\end{aligned}
$$

BULlETIN DE LA SOCIÉTÉ MATHÉMATIQUE DE FRANCE 
D'autre part, pour $s \in \mathbb{C}$ vérifiant $\sigma>1$ et $|\tau| \geq 1$, les relations (17) et (18) appliquées avec $N=\sup ([n+1-\sigma], 0) \geq 0$ entraînent que pour tout $\varepsilon>0$,

$$
\begin{aligned}
Z_{\text {proj }}^{*}(P ; s) & \ll_{\sigma} \sum_{|\alpha| \leq N-1}\left|\left(\begin{array}{c}
-s / d \\
|\alpha|
\end{array}\right)\right| \cdot\left|Z\left(P_{d}, T_{\alpha} ; \frac{s}{d}+|\alpha|\right)\right|+|\tau|^{N+1} \\
& \ll_{\sigma, \varepsilon}|\tau|^{n+1-\sigma+\varepsilon}+|\tau|^{N+1} .
\end{aligned}
$$

On en déduit que l'on a $Z_{\text {proj }}^{*}(P ; s) \ll_{\sigma, \varepsilon} 1+|\tau|^{n+2-\sigma+\varepsilon}(\sigma>1$ et $|\tau| \geq 1)$ pour tout $\varepsilon>0$.

Soit maintenant $\varepsilon>0$ fixé et posons $\delta=\frac{1}{2} \varepsilon>0$. Le choix $N=n-1$ dans $\left(^{*}\right)$ montre que $Z_{\text {proj }}^{*}(P ; 1+\delta+i \tau) \ll_{\delta}|\tau|^{n+\delta}$ (où $|\tau| \geq 1$ ). De plus il est clair que $Z_{\text {proj }}^{*}(P ; n+1+\delta+i \tau) \ll_{\delta} 1 \ll_{\delta}|\tau|^{0}$ (où $|\tau| \geq 1$ ).

On en déduit d'après le thèorème classique de Phragmén-Lindelof (voir [17, p. 123]), que $Z_{\text {proj }}^{*}(P ; s) \ll_{\delta} 1+|\tau|^{k(\sigma)}($ avec $\sigma \in[1+\delta, n+1+\delta]$ et $|\tau| \geq 1$ ) où $k(\sigma)$ est la fonction affine prenant respectivement les valeurs 0 et $n+\delta$ en $n+1+\delta$ et $1+\delta$. Un calcul facile montre alors que l'on a

$$
k(\sigma)=n+1-\sigma+\varepsilon+\frac{\delta(1+\delta-\sigma)}{n} \leq n+1-\sigma+\varepsilon
$$

pour tout $\sigma \in[1+\delta, n+1+\delta]$. Cela termine la preuve de la partie du théorème 2.3 concernant $Z_{\text {proj }}^{*}(P ; s)$.

4.2.2. Étude de $Z_{\text {proj }}(P ; s)$. - Comme dans l'étude de $Z_{n, \infty}(s)$ (paragraphe 4.1.2), nous posons pour tout $I \subset\{0, \ldots, n\}$,

$$
V_{n}^{I}=\left\{\left(x_{0}: \ldots: x_{n}\right) \in \mathbb{P}_{n}(\mathbb{Q}) \mid x_{i} \neq 0, \forall i \in I \text { et } x_{i}=0, \forall i \notin I\right\} .
$$

On obtient, vu l'étude de $Z_{\text {proj }}^{*}(P ; s)$, que pour tout $s \in \mathbb{C}$ vérifiant $\operatorname{Re}(s)>n+1$

$$
\begin{aligned}
Z_{\text {proj }}(P ; s) & =\sum_{\varnothing \neq I \subset\{0, \ldots, n\}} \sum_{M \in V_{n}^{I}(\mathbb{Q})} \frac{1}{H_{P}^{s}(M)} \\
& =\sum_{r=0}^{n} \sum_{0 \leq i_{0}<\cdots<i_{r} \leq n} \sum_{M \in V_{n}^{\left\{i_{0}, \ldots, i_{r}\right\}}(\mathbb{Q})} \frac{1}{H_{P}^{s}(M)} .
\end{aligned}
$$

D'autre part, pour tout $r=0, \ldots, n$ et pour tout $0 \leq i_{0}<\cdots<i_{r} \leq n$, définissons $P_{i_{0}, \ldots, i_{r}}$ le polynôme de $\mathbb{R}\left[X_{0}, \ldots, X_{r}\right]$ tel que

$$
P_{i_{0}, \ldots, i_{r}}\left(X_{0}, \ldots, X_{r}\right)=P\left(Y_{0}, \ldots, Y_{n}\right)
$$

avec pour tout $j=0, \ldots, n, Y_{j}=X_{k}$ s'il existe $k \in\{0, \ldots, r\}$ tel que $j=i_{k}$, et $Y_{j}=0$ sinon. Alors pour tout $s \in \mathbb{C}$ vérifiant $\operatorname{Re}(s)>n+1$,

$$
Z_{\text {proj }}(P ; s)=\sum_{r=0}^{n} \sum_{0 \leq i_{0}<\cdots<i_{r} \leq n} Z_{\text {proj }}^{*}\left(P_{i_{0}, \ldots, i_{r}} ; s\right) .
$$


Comme $P_{i_{0}, \ldots, i_{r}}$ est elliptique pour tout $i_{0}, \ldots, i_{r}$ tel que $0 \leq i_{0}<\cdots<i_{r} \leq n$, alors la relation (19) et le résultat de la section précédente terminent la preuve du théorème 2.3 .

4.2.3. Preuve du corollaire 2.4. - Dans le cas où le polynôme $P$ est elliptique, le corollaire 2.4 découle immédiatement de la proposition 3.1 qu'on applique aux deux séries $s \mapsto Z_{\text {proj }}(P ; s)$ et $s \mapsto Z_{\text {proj }}^{*}(P ; s)$ en prenant $\sigma_{0}=n+1$, $A=1, \delta=n$ et donc $\mu=\sup \left(\delta^{-1}, A\right)=1$.

Dans le cas plus particulier où $P$ est homogène, on commence par appliquer la proposition 3.1 (point 3 de la conclusion) aux séries $s \mapsto \zeta(s) Z_{\text {proj }}(P ; s)$ et $s \mapsto \zeta(s) Z_{\text {proj }}^{*}(P ; s)$; on conclut ensuite par sommation d'Abel. Dans les deux cas, le théorème 2.3 permet de vérifier les hypothèses.

\section{Preuve des théorèmes concernant le plan projectif éclaté}

On reprend les notations du paragraphe 1.2.2 :

$$
X=X(\mathbb{Q})=\left\{((x: y: z),(u: v)) \in \mathbb{P}_{2}(\mathbb{Q}) \times \mathbb{P}_{1}(\mathbb{Q}) \mid x v-y u=0\right\} .
$$

On pose

$$
\begin{aligned}
& V_{1}=\{((x: y: z),(u: v)) \in X \mid x y z \neq 0\}, \\
& V_{2}=\{((x: y: z),(u: v)) \in X \mid x=0, y z \neq 0\} \\
& =\left\{((0: y: z),(0: 1)) \mid(y: z) \in \mathbb{P}_{1}(\mathbb{Q}), y z \neq 0\right\}, \\
& V_{3}=\{((x: y: z),(u: v)) \in X \mid x=0, y=0, z \neq 0\} \\
& =\{(0: 0: 1)\} \times \mathbb{P}_{1}=E, \\
& V_{4}=\{((x: y: z),(u: v)) \in X \mid x=0, y \neq 0, z=0\} \\
& =\{((0: 1: 0),(0: 1))\}, \\
& V_{5}=\{((x: y: z),(u: v)) \in X \mid x y \neq 0, z=0\} \\
& =\left\{((x: y: 0),(x: y)) \mid(x: y) \in \mathbb{P}_{1}(\mathbb{Q}), x y \neq 0\right\}, \\
& V_{6}=\{((x: y: z),(u: v)) \in X \mid x \neq 0, y=0, z=0\} \\
& =\{((1: 0: 0),(1: 0))\}, \\
& V_{7}=\{((x: y: z),(u: v)) \in X \mid x z \neq 0, y=0\} \text {, } \\
& =\left\{((x: 0: z),(1: 0)) \mid(x: z) \in \mathbb{P}_{1}(\mathbb{Q}), x z \neq 0\right\} .
\end{aligned}
$$

On a évidement les réunions disjointes

$$
X=\bigcup_{j=1, \ldots, 7}^{\bullet} V_{j} \text { et } U=X \backslash E=X \backslash V_{3}=\bigcup_{\substack{j=1, \ldots, 7 \\ j \neq 3}}^{\bullet} V_{j} .
$$

BULlETin DE LA SOCiÉtÉ MATHÉMATIQUe DE FRANCE 
On considère pour tout $j=1, \ldots, 7$ et tout $i \in\{\infty, 2\}$ :

$$
Z_{V_{j}}^{i}\left(\mathcal{L}_{a, b} ; s\right)=\sum_{P \in V_{j}(\mathbb{Q})}\left(H_{a, b}^{i}\right)^{-s}(P) .
$$

Comme les hauteurs $H_{a, b}^{i}$ sont symétriques en $x, y, z$ et en $u, v$, il est clair que $Z_{V_{2}}^{i}\left(\mathcal{L}_{a, b} ; s\right)=Z_{V_{7}}^{i}\left(\mathcal{L}_{a, b} ; s\right)$ et $Z_{V_{4}}^{i}\left(\mathcal{L}_{a, b} ; s\right)=Z_{V_{6}}^{i}\left(\mathcal{L}_{a, b} ; s\right) \equiv 1$. Ceci entraîne alors que, pour tout $a, b \in \mathbb{Z}$ tels que $a>0$ et $b>0$, tout $i \in\{\infty, 2\}$ et tout $s$ dans le domaine de convergence absolue de $Z_{X}^{i}\left(\mathcal{L}_{a, b} ; s\right)$, on a

$$
\begin{aligned}
& Z_{X}^{i}\left(\mathcal{L}_{a, b} ; s\right)=\sum_{j=1}^{7} Z_{V_{j}}^{i}\left(\mathcal{L}_{a, b} ; s\right) \\
& \quad=Z_{V_{1}}^{i}\left(\mathcal{L}_{a, b} ; s\right)+2 Z_{V_{2}}^{i}\left(\mathcal{L}_{a, b} ; s\right)+Z_{V_{3}}^{i}\left(\mathcal{L}_{a, b} ; s\right)+Z_{V_{5}}^{i}\left(\mathcal{L}_{a, b} ; s\right)+2 .
\end{aligned}
$$

De même, pour tout $a, b \in \mathbb{Z}$ tels que $a>0$ et $a+b>0$, tout $i \in\{\infty, 2\}$ et tout $s$ dans le domaine de convergence absolue de $Z_{U}^{i}\left(\mathcal{L}_{a, b} ; s\right)$, on a

$$
\begin{aligned}
& Z_{U}^{i}\left(\mathcal{L}_{a, b} ; s\right)=\sum_{\substack{j=1, \ldots, 7 \\
j \neq 3}} Z_{V_{j}}^{i}\left(\mathcal{L}_{a, b} ; s\right) \\
& \quad=Z_{V_{1}}^{i}\left(\mathcal{L}_{a, b} ; s\right)+2 Z_{V_{2}}^{i}\left(\mathcal{L}_{a, b} ; s\right)+Z_{V_{5}}^{i}\left(\mathcal{L}_{a, b} ; s\right)+2 .
\end{aligned}
$$

En particulier pour tout $a, b \in \mathbb{N}^{*}$ et pour $\operatorname{Re}(s)$ assez grande, on a

$$
Z_{X}^{i}\left(\mathcal{L}_{a, b} ; s\right)=Z_{U}^{i}\left(\mathcal{L}_{a, b} ; s\right)+Z_{V_{3}}^{i}\left(\mathcal{L}_{a, b} ; s\right) .
$$

\section{1. Étude des termes: $Z_{V_{j}}^{i}\left(\mathcal{L}_{a, b} ; s\right)(i=\infty, 2$ et $j=1,2,3,5)$}

5.1.1. Étude de $Z_{V_{j}}^{i}\left(\mathcal{L}_{a, b} ; s\right)(i=\infty, 2$ et $j=2,3,5)$. — Les symétries des hauteurs et la proposition 3.8, impliquent facilement que

(23) si $a, b>0$, alors pour $\operatorname{Re}(s)>2 / b$, on a $Z_{V_{3}}^{i}\left(\mathcal{L}_{a, b} ; s\right)=Z_{1, i}(b s)$;

(24) si $a, a+b>0$, alors pour $\operatorname{Re}(s)>2 / a$, on a $Z_{V_{2}}^{i}\left(\mathcal{L}_{a, b} ; s\right)=Z_{1, i}^{*}(a s)$;

(25) si $a, a+b>0$, alors pour $\operatorname{Re}(s)>2 /(a+b)$, on a

$$
Z_{V_{5}}^{i}\left(\mathcal{L}_{a, b} ; s\right)=Z_{1, i}^{*}((a+b) s) .
$$

5.1.2. Étude de $Z_{V_{1}}^{\infty}\left(\mathcal{L}_{a, b} ; s\right)(i=\infty, 2)$ dans le cas $a>0$ et $a+b>0$. Rappelons que

$$
\begin{aligned}
V_{1} & =\{((x: y: z),(u: v)) \in X \mid x y z \neq 0\} \\
& =\left\{((x: y: z),(x: y)) \mid(x: y: z) \in \mathbb{P}_{2}, x y z \neq 0\right\} .
\end{aligned}
$$

Comme $a+b>0$ et $a>0$, il est alors clair que pour tout $(x: y: z) \in \mathbb{P}_{2}$, on a

$$
\begin{aligned}
H_{a, b}^{\infty}((x: y: z),(x: y)) & =\left(H_{\infty}^{2}(x: y: z)\right)^{a}\left(H_{\infty}^{1}(x: y)\right)^{b} \\
& \geq\left(H_{\infty}^{2}(x: y: z)\right)^{\inf (a, a+b)} .
\end{aligned}
$$

TOME $133-2005-\mathrm{N}^{\mathrm{O}} 2$ 
On en déduit que $s \mapsto Z_{V_{1}}^{\infty}\left(\mathcal{L}_{a, b} ; s\right)$ converge absolument dans le demi-plan $\{\operatorname{Re}(s)>c=3 / \inf (a, a+b)\}$ et y vérifie

$$
\begin{aligned}
Z_{V_{1}}^{\infty}\left(\mathcal{L}_{a, b} ; s\right) & =4 \sum_{\substack{(x, y, z) \in \mathbb{N}^{* 3} \\
\operatorname{pgcd}(x, y, z)=1}} \frac{1}{\sup (x, y, z)^{a s}[\sup (n(x, y), n(y, x))]^{b s}} \\
& =\frac{4}{\zeta(a s)} \sum_{(x, y, z) \in \mathbb{N}^{* 3}} \frac{1}{\sup (x, y, z)^{a s}[\sup (n(x, y), n(y, x))]^{b s}} .
\end{aligned}
$$

La dernière égalité découle du lemme 3.3.

En utilisant la partition

$$
\begin{aligned}
\mathbb{N}^{* 3}= & \left\{(x, y, z) \in \mathbb{N}^{* 3} \mid x>y>z\right\} \dot{\cup}\{y>x>z\} \dot{\cup}\{z>x>y\} \dot{\cup}\{z>y>x\} \\
& \dot{\cup}\{x>z>y\} \dot{\cup}\{y>z>x\} \dot{\cup}\{x=y>z\} \dot{\cup}\{z>x=y\} \dot{\cup}\{z=x>y\} \\
& \dot{\cup}\{y>x=z\} \dot{\cup}\{z=y>x\} \dot{\cup}\{x>z=y\} \dot{\cup}\{x=y=z\}
\end{aligned}
$$

et tenant compte des symétries des domaines et de la hauteur, on obtient pour $\operatorname{Re}(s)>c:$

$$
\begin{aligned}
Z_{V_{1}}^{\infty}\left(\mathcal{L}_{a, b} ; s\right)=\frac{8}{\zeta(a s)} \sum_{x>y>z \geq 1} \frac{1}{x^{a s}(n(x, y))^{b s}}+\frac{8}{\zeta(a s)} \sum_{z>x>y \geq 1} \frac{1}{z^{a s}(n(x, y))^{b s}} \\
+\frac{8}{\zeta(a s)} \sum_{x>z>y \geq 1} \frac{1}{x^{a s}(n(x, y))^{b s}}+\frac{16}{\zeta(a s)} \sum_{x>y \geq 1} \frac{1}{x^{a s}(n(x, y))^{b s}} \\
\quad+\frac{8}{\zeta(a s)} \sum_{x>z \geq 1} \frac{1}{x^{a s}}+4 .
\end{aligned}
$$

On en déduit alors que pour $\operatorname{Re}(s)>c$, on a

$$
\begin{aligned}
Z_{V_{1}}^{\infty}\left(\mathcal{L}_{a, b} ; s\right)= & \frac{8}{\zeta(a s)} \sum_{x>y \geq 1} \frac{x}{x^{a s}(n(x, y))^{b s}} \\
& +\frac{8}{\zeta(a s)} \sum_{z>x>y \geq 1} \frac{1}{z^{a s}(n(x, y))^{b s}}+8 \frac{\zeta(a s-1)}{\zeta(a s)}-4 \\
= & \frac{8}{\zeta(a s)} \sum_{x \geq y \geq 1} \frac{1}{x^{a s-1}(n(x, y))^{b s}} \\
& +\frac{8}{\zeta(a s)} \sum_{z>x>y \geq 1} \frac{1}{z^{a s}(n(x, y))^{b s}}-4
\end{aligned}
$$

Or le lemme 3.4 implique

$$
\sum_{x \geq y} \frac{1}{x^{a s-1}(n(x, y))^{b s}}=\frac{\zeta(a s-1) \zeta((a+b) s-2)}{\zeta((a+b) s-1)} .
$$

BULletin DE LA SOCiÉtÉ MATHÉMATIQUe DE FRANCE 
L'équation (26) entraîne alors que pour $\operatorname{Re}(s)>c$, on a

$$
\begin{gathered}
Z_{V_{1}}^{\infty}\left(\mathcal{L}_{a, b} ; s\right)=8 \frac{\zeta(a s-1) \zeta((a+b) s-2)}{\zeta(a s) \zeta((a+b) s-1)}-4+\frac{8}{\zeta(a s)} M(s) \\
\text { où } M(s)=\sum_{z>x>y \geq 1} \frac{1}{z^{a s}(n(x, y))^{b s}} .
\end{gathered}
$$

On fixe maintenant $N \in \mathbb{N}$ quelconque; le lemme 3.5 implique alors :

$$
\begin{aligned}
& M(s)= \sum_{z>x>y \geq 1} \frac{1}{z^{a s}(n(x, y))^{b s}}=\sum_{x>y} \frac{1}{(n(x, y))^{b s}}\left(\sum_{z>x} \frac{1}{z^{a s}}\right) \\
&= \sum_{x>y} \frac{1}{(n(x, y))^{b s}}\left\{\frac{1}{(a s-1)} \frac{1}{x^{a s-1}}\right. \\
&\left.\quad+\sum_{k=0}^{N} \frac{(-1)^{k} B_{k+1}}{(k+1) x^{a s+k}}\left(\begin{array}{c}
-a s \\
k
\end{array}\right)+R_{N}(x ; a s)\right\} \\
&=\frac{1}{a s-1} \sum_{x>y} \frac{1}{x^{a s-1}(n(x, y))^{b s}} \\
& \quad+\sum_{k=0}^{N} \frac{(-1)^{k} B_{k+1}}{k+1}\left(\begin{array}{c}
-a s \\
k
\end{array}\right)\left(\sum_{x>y} \frac{1}{x^{a s+k}(n(x, y))^{b s}}\right) \\
& \quad+\sum_{k=0}^{N} \frac{(-1)^{k} B_{k+1}}{k+1}\left(\begin{array}{c}
-a s \\
k
\end{array}\right)\left(\sum_{x \geq y} \frac{1}{x^{a s+k}(n(x, y))^{b s}}\right) \\
& \quad+\sum_{x>y} \frac{R_{N}(x ; a s)}{(n(x, y))^{b s}}-\frac{\zeta(a s-1)}{a s-1}-\sum_{k=0}^{N} \frac{(-1)^{k} B_{k+1}}{k+1}\left(\begin{array}{c}
-a s \\
k
\end{array}\right) \zeta(a s+k) .
\end{aligned}
$$

Le lemme 3.4 implique alors que pour $\operatorname{Re}(s)>c$, on a

$$
\begin{aligned}
& M(s)=\frac{\zeta(a s-1) \zeta((a+b) s-2)}{(a s-1) \zeta((a+b) s-1)} \\
& +\sum_{k=0}^{N} \frac{(-1)^{k} B_{k+1}}{k+1}\left(\begin{array}{c}
-a s \\
k
\end{array}\right) \frac{\zeta(a s+k) \zeta((a+b) s+k-1)}{\zeta((a+b) s+k)} \\
& -\frac{\zeta(a s-1)}{a s-1}-\sum_{k=0}^{N} \frac{(-1)^{k} B_{k+1}}{k+1}\left(\begin{array}{c}
-a s \\
k
\end{array}\right) \zeta(a s+k)+\sum_{x>y} \frac{R_{N}(x ; a s)}{(n(x, y))^{b s}} .
\end{aligned}
$$


En remarquant qu'uniformément en $s=\sigma+i \tau$ vérifiant $\sigma>-N / a$

$$
\sum_{x>y}\left|\frac{R_{N}(x ; a s)}{(n(x, y))^{b s}}\right| \ll_{N, \sigma}\left(1+|\tau|^{N+1}\right) \sum_{x \geq y} \frac{1}{x^{a \sigma+N}} \frac{1}{(n(x, y))^{b \sigma}},
$$

le lemme 3.4 implique alors que pour tout $s=\sigma+i \tau$ vérifiant la condition $\sigma>\sup ((1-N) / a,(2-N) /(a+b))$, on a

$$
\begin{aligned}
\sum_{x>y}\left|\frac{R_{N}(x ; a s)}{(n(x, y))^{b s}}\right| & \ll_{N, \sigma}\left(1+|\tau|^{N+1}\right) \frac{\zeta(a \sigma+N) \zeta((a+b) \sigma+N-1)}{\zeta((a+b) \sigma+N)} \\
& \ll_{N, \sigma} 1+|\tau|^{N+1} .
\end{aligned}
$$

Les relations (27), (28), (29) et (30) impliquent alors la proposition suivante :

Proposition 5.1. - Soit $N \in \mathbb{N}$, alors pour tout $s \in \mathbb{C}$ tel que $\operatorname{Re}(s)>c$. Alors on a

$$
\begin{aligned}
& Z_{V_{1}}^{\infty}\left(\mathcal{L}_{a, b} ; s\right)=8 \frac{a s \zeta(a s-1) \zeta((a+b) s-2)}{(a s-1) \zeta(a s) \zeta((a+b) s-1)}-8 \frac{\zeta(a s-1)}{(a s-1) \zeta(a s)} \\
&+8 \sum_{k=0}^{N} \frac{(-1)^{k} B_{k+1}}{k+1}\left(\begin{array}{c}
-a s \\
k
\end{array}\right) \frac{\zeta(a s+k) \zeta((a+b) s+k-1)}{\zeta(a s) \zeta((a+b) s+k)} \\
&-8 \sum_{k=0}^{N} \frac{(-1)^{k} B_{k+1}}{k+1}\left(\begin{array}{c}
-a s \\
k
\end{array}\right) \frac{\zeta(a s+k)}{\zeta(a s)}+\frac{M_{N}(s)}{\zeta(a s)}-4
\end{aligned}
$$

où $s \mapsto M_{N}(s)$ est holomorphe dans le demi-plan

$$
\left\{\operatorname{Re}(s)>\sup \left(\frac{1-N}{a}, \frac{2-N}{a+b}\right)\right\}
$$

et qui y vérifie $M_{N}(s) \ll_{N, \operatorname{Re}(s)} 1+|\tau|^{N+1}$.

En particulier, comme $N$ peut être arbitrairement grand, il découle de cette proposition que $s \mapsto Z_{V_{1}}^{\infty}\left(\mathcal{L}_{a, b} ; s\right)$ possède un prolongement méromorphe à tout le plan complexe avec des pôles contenus dans l'ensemble

$$
\left\{\frac{2}{a}, \frac{3-k}{a+b}, \frac{\beta}{a}, \frac{1+\beta-k}{a+b} \mid k \in \mathbb{N}, \beta \in \mathcal{Z}(\zeta)\right\} .
$$

5.1.3. Étude de $Z_{V_{1}}^{2}\left(\mathcal{L}_{a, b} ; s\right)$ dans le cas $a>0$ et $a+b>0$. - On démontre de la même façon que dans le cas de $Z_{V_{1}}^{\infty}\left(\mathcal{L}_{a, b} ; s\right)$ que $s \mapsto Z_{V_{1}}^{2}\left(\mathcal{L}_{a, b} ; s\right)$ converge absolument dans le demi-plan $\operatorname{Re}(s)>c=3 / \sup (a, a+b)$. On a aussi, pour 
les mêmes raisons et en vertu du lemme 3.3 , que pour $\operatorname{Re}(s)>c$, on a

$$
\begin{aligned}
& Z_{V_{1}}^{2}\left(\mathcal{L}_{a, b} ; s\right)=4 \sum_{\substack{(x, y, z) \in \mathbb{N}^{* 3} \\
\operatorname{pgcd}(x, y, z)=1}} H_{a, b}^{2}((x: y: z),(x: y))^{-s} \\
& =4 \sum_{\substack{(x, y, z) \in \mathbb{N}^{* 3} \\
\operatorname{pgcd}(x, y, z)=1}} \frac{1}{{\sqrt{x^{2}+y^{2}+z^{2}}}^{a s}{\sqrt{(n(x, y))^{2}+(n(y, x))^{2}}}^{b s}} \\
& =\frac{4}{\zeta(a s)} \sum_{(x, y, z) \in \mathbb{N}^{* 3}} \frac{1}{{\sqrt{x^{2}+y^{2}+z^{2}}}^{a s}{\sqrt{(n(x, y))^{2}+(n(y, x))^{2}}}^{b s}} \\
& =\frac{4}{\zeta(a s)} \sum_{x, y \geq 1} \frac{1}{\sqrt{(n(x, y))^{2}+(n(y, x))^{2}} b s} F\left(\sqrt{x^{2}+y^{2}} ; a s\right)
\end{aligned}
$$

où $F(\alpha ; s)=\sum_{z \geq 1}\left(\alpha^{2}+z^{2}\right)^{-s / 2}$ est la fonction etudiée dans le lemme 3.6.

Fixons maintenant un entier $N \in \mathbb{N}$ quelconque. Le lemme 3.6 entraîne

$$
F(\alpha ; s)=\frac{\sqrt{\pi} \Gamma\left(\frac{1}{2}(s-1)\right)}{2 \alpha^{s-1} \Gamma\left(\frac{1}{2} s\right)}-\frac{1}{2 \alpha^{s}}+R(\alpha ; s)
$$

où $s \mapsto R(\alpha ; s)$ est une fonction entière qui vérifie, pour tout $\sigma>-N$,

$$
R(\alpha ; s) \ll_{N, \sigma} \frac{1+|\tau|^{N+1}}{\alpha^{\sigma+N}} \quad \text { uniformément en } \tau \in \mathbb{R} \text { et en } \alpha \geq 1 .
$$

Les relations (31) et (32) impliquent alors que pour $\operatorname{Re}(s)>c$, on a

$$
\begin{aligned}
& Z_{V_{1}}^{2}\left(\mathcal{L}_{a, b} ; s\right) \\
& =\frac{2 \sqrt{\pi} \Gamma\left(\frac{1}{2}(a s-1)\right)}{\Gamma\left(\frac{1}{2} a s\right) \zeta(a s)} \sum_{x, y \geq 1} \frac{1}{{\sqrt{x^{2}+y^{2}}}^{a s-1}} \frac{1}{{\sqrt{(n(x, y))^{2}+(n(y, x))^{2}}}^{b s}} \\
& -\frac{2}{\zeta(a s)} \sum_{x, y \geq 1} \frac{1}{{\sqrt{x^{2}+y^{2}}}^{a s}} \frac{1}{{\sqrt{(n(x, y))^{2}+(n(y, x))^{2}}}^{b s}} \\
& +\frac{4}{\zeta(a s)} \sum_{x, y \geq 1} \frac{R\left(\sqrt{x^{2}+y^{2}} ; a s\right)}{{\sqrt{(n(x, y))^{2}+(n(y, x))^{2}}}^{b s}} .
\end{aligned}
$$

Le lemme 3.9 implique alors que

$$
\begin{array}{r}
Z_{V_{1}}^{2}\left(\mathcal{L}_{a, b} ; s\right)=\frac{2 \sqrt{\pi} \Gamma\left(\frac{1}{2}(a s-1)\right) \zeta(a s-1) G((a+b) s-1)}{\Gamma\left(\frac{1}{2} a s\right) \zeta(a s) \zeta((a+b) s-1)} \\
-\frac{2 G((a+b) s)}{\zeta((a+b) s)}+\frac{F(s)}{\zeta(a s)}
\end{array}
$$

où

$$
F(s)=4 \sum_{x, y \geq 1} \frac{R\left(\sqrt{x^{2}+y^{2}} ; a s\right)}{{\sqrt{(n(x, y))^{2}+(n(y, x))^{2}}}^{b s}} .
$$


La majoration uniforme de $R(\alpha ; s)$ donnée par (33) et le lemme 3.9 permettent de voir facilement que $s \mapsto F(s)$ est holomorphe dans le demi-plan $\{\operatorname{Re}(s)>$ $\sup ((1-N) / a,(2-N) /(a+b))\}$ et y vérifie $F(\sigma+i \tau) \ll_{N, \sigma}\left(1+|\tau|^{N+1}\right)$.

En faisant tendre $N$ vers l'infini, on obtient alors :

Proposition 5.2. - Pour tout $s \in \mathbb{C}$ de partie réelle assez grande, on a

$$
\begin{array}{r}
Z_{V_{1}}^{2}\left(\mathcal{L}_{a, b} ; s\right)=\frac{2 \sqrt{\pi} \Gamma\left(\frac{1}{2}(a s-1)\right) \zeta(a s-1) G((a+b) s-1)}{\Gamma\left(\frac{1}{2} a s\right) \zeta(a s) \zeta((a+b) s-1)} \\
-\frac{2 G((a+b) s)}{\zeta((a+b) s)}+\frac{F(s)}{\zeta(a s)}
\end{array}
$$

où $s \mapsto F(s)$ est une fonction entière qui vérifiee $F(s) \ll_{N, \sigma} 1+|\tau|^{N+1}$ pour tout $N \in \mathbb{N}$, uniformément en $s=\sigma+i \tau$ tel que

$$
\sigma>\sup \left(\frac{1-N}{a}, \frac{2-N}{a+b}\right)
$$

En particulier, il découle de cette proposition que $s \mapsto Z_{V_{1}}^{2}\left(\mathcal{L}_{a, b} ; s\right)$ possède un prolongement méromorphe à tout le plan complexe avec des pôles contenus dans l'ensemble

$$
\left\{\frac{2}{a}, \frac{3}{a+b}, \frac{2}{a+b}, \frac{1}{a+b}\right\} \cup\left\{\frac{1-2 k}{a}, \frac{\beta}{a}, \frac{1+\beta}{a+b}, \frac{\beta}{a+b} \mid k \in \mathbb{N}, \beta \in \mathcal{Z}(\zeta)\right\} .
$$

5.2. Preuve des théorèmes 2.5 et 2.6 pour $i=\infty$. - Dans ce paragraphe, $a$ et $b$ sont deux entiers relatifs tels que $a>0$ et $a+b>0$. D'après les relations (21), (24) et (25), pour tout $s \in \mathbb{C}$ de partie réelle assez grande, on a

$$
Z_{U}^{\infty}\left(\mathcal{L}_{a, b} ; s\right)=Z_{V_{1}}^{\infty}\left(\mathcal{L}_{a, b} ; s\right)+2 Z_{1, \infty}^{*}(a s)+Z_{1, \infty}^{*}((a+b) s)+2 .
$$

Or la proposition 3.8 implique $Z_{1, \infty}^{*}(s)=4 \zeta(s-1) /(\zeta(s))-2$. On en déduit alors que

$$
Z_{U}^{\infty}\left(\mathcal{L}_{a, b} ; s\right)=Z_{V_{1}}^{\infty}\left(\mathcal{L}_{a, b} ; s\right)+\frac{8 \zeta(a s-1)}{\zeta(a s)}+\frac{4 \zeta((a+b) s-1)}{\zeta((a+b) s)}-4 .
$$

Le théorème 2.5 pour $i=\infty$ découle alors de cette dernière relation, de la proposition 5.1 et des propriétés classiques de la fonction zêta de Riemann.

Le théorème 2.6 s'obtient en appliquant la proposition 5.1 avec $N=0$. En effet, pour $N=0$, cette proposition donne

$$
\begin{aligned}
Z_{V_{1}}^{\infty}\left(\mathcal{L}_{a, b} ; s\right)= & 8 \frac{a s}{a s-1} \frac{\zeta(a s-1) \zeta((a+b) s-2)}{\zeta(a s) \zeta((a+b) s-1)} \\
& -4 \frac{\zeta((a+b) s-1)}{\zeta((a+b) s)}-8 \frac{\zeta(a s-1)}{(a s-1) \zeta(a s)}+\frac{M_{0}(s)}{\zeta(a s)}
\end{aligned}
$$

BULlETIN DE LA SOCIÉTÉ MATHÉMATIQUE DE FRANCE 
où $s \mapsto M_{0}(s)$ est une fonction holomorphe dans $\{\operatorname{Re}(s)>\sup (1 / a, 2 /(a+b))\}$ et qui y vérifie $M_{0}(s) \ll_{\sigma} 1+|\tau|$. Ainsi, pour $\operatorname{Re}(s)$ assez grande :

$$
\begin{aligned}
Z_{U}^{\infty}\left(\mathcal{L}_{a, b} ; s\right)=8 \frac{a s \zeta(a s-1) \zeta((a+b) s-2)}{(a s-1) \zeta(a s) \zeta((a+b) s-1)} & \\
& +8 \frac{(a s-2) \zeta(a s-1)}{(a s-1) \zeta(a s)}+\frac{M_{0}(s)}{\zeta(a s)}-4 .
\end{aligned}
$$

Le premier pôle est alors $\sup (2 / a, 3 /(a+b))$; c'est un pôle simple si $a \neq 2 b$ et double sinon. La détermination de la partie polaire est un simple calcul de résidu utilisant que la partie polaire de $\zeta$ au point $s=1$ est donnée par $\zeta(s)=1 /(s-1)+\gamma+\mathcal{O}(|s-1|)$ quand $s$ tend vers 1.

On remarque enfin, que le plus grand pôle $\alpha$ est aussi l'abscisse de convergence absolue de la série à termes positifs $s \mapsto Z_{U}^{\infty}\left(\mathcal{L}_{a, b} ; s\right)$ d'après le théorème de Landau (voir [9] ou [17, cor. 6.1, p. 113]). Cela termine la preuve des théorèmes 2.5 et 2.6 pour $i=\infty$.

5.3. Fin des preuves des théorèmes 2.5 et 2.6 pour $\boldsymbol{i}=\mathbf{2}$. - Dans ce paragraphe, $a$ et $b$ sont deux entiers relatifs tels que $a>0$ et $a+b>0$. D'après les relations (21), (24) et (25), pour tout $s \in \mathbb{C}$ de partie réelle assez grande,

$$
Z_{U}^{2}\left(\mathcal{L}_{a, b} ; s\right)=Z_{V_{1}}^{2}\left(\mathcal{L}_{a, b} ; s\right)+2 Z_{1,2}^{*}(a s)+Z_{1,2}^{*}((a+b) s)+2 .
$$

Or la proposition 3.8 implique $Z_{1,2}^{*}(s)=2 G(s) / \zeta(s)$. On en déduit alors que

$$
Z_{U}^{2}\left(\mathcal{L}_{a, b} ; s\right)=Z_{V_{1}}^{2}\left(\mathcal{L}_{a, b} ; s\right)+\frac{4 G(a s)}{\zeta(a s)}+\frac{2 G((a+b) s)}{\zeta((a+b) s)}+2
$$

où $G(s)=\sum_{k, \ell \geq 1}\left(k^{2}+\ell^{2}\right)^{-s / 2}$ est la fonction étudiée dans le lemme 3.7.

Le théorème 2.5 pour $i=2$ découle alors de cette dernière relation, de la proposition 5.2, du lemme 3.7 et des propriétés classiques de la fonction zêta de Riemann.

Le théorème 2.6 s'obtient en appliquant la proposition 5.2 pour $N=0$. En effet, cette proposition donne

$$
\begin{array}{r}
Z_{V_{1}}^{2}\left(\mathcal{L}_{a, b} ; s\right)=\frac{2 \sqrt{\pi} \Gamma\left(\frac{1}{2}(a s-1)\right) \zeta(a s-1) G((a+b) s-1)}{\Gamma\left(\frac{1}{2} a s\right) \zeta(a s) \zeta((a+b) s-1)} \\
-\frac{2 G((a+b) s)}{\zeta((a+b) s)}+\frac{F(s)}{\zeta(a s)}
\end{array}
$$

où $s \mapsto F(s)$ est une fonction entière holomorphe qui vérifie $F(s) \ll_{\sigma} 1+$ $|\tau|$ dans le demi-plan $\{\operatorname{Re}(s)>\sup (1 / a, 2 /(a+b))\}$. Ainsi, pour $\operatorname{Re}(s)$ assez 
grande

$$
\begin{array}{r}
Z_{U}^{2}\left(\mathcal{L}_{a, b} ; s\right)=\frac{2 \sqrt{\pi} \Gamma\left(\frac{1}{2}(a s-1)\right) \zeta(a s-1) G((a+b) s-1)}{\Gamma\left(\frac{1}{2} a s\right) \zeta(a s) \zeta((a+b) s-1)} \\
+\frac{4 G(a s)}{\zeta(a s)}+2+\frac{F(s)}{\zeta(a s)} .
\end{array}
$$

Le lemme 3.7 et les propriétés classiques des fonctions zêta de Riemann et Gamma d'Euler permettent de conclure de la même façon que dans le paragraphe 5.2.

\section{Preuve du corollaire 2.7}

Le corollaire 2.7 découle du théorème 2.6 par application de la proposition 3.1. Nous allons néanmoins détailler le cas le plus important à savoir $a=2 b>0$ et ceci pour $i=\infty$ (le cas $i=2$ se traite de façon similaire).

Détails des calculs conduisant aux parties 2) et 3) du théorème 2.6 et preuve $d u$ corollaire 2.7 dans le cas $a=2 b>0$ et $i=\infty$. - Nous utiliserons les notations du théorème 2.6 et de la proposition 3.1. Comme $a=2 b$, on a alors $\alpha=2 / a=3 /(a+b)=1 / b$ et $\alpha^{\prime}=2 / 3 b=\frac{2}{3} \alpha$.

D'après la partie 1) du théorème 2.6 , on sait que pour $\sigma>\alpha$, on a

$$
\begin{aligned}
Z_{U}^{\infty}(s) & :=Z_{U}^{\infty}\left(\mathcal{L}_{a, b} ; s\right)=Z_{U}^{\infty}\left(\mathcal{L}_{2 b, b} ; s\right) \\
& =\frac{16 b s \zeta(2 b s-1) \zeta(3 b s-2)}{(2 b s-1) \zeta(2 b s) \zeta(3 b s-1)}+R_{U}^{\infty}(s)
\end{aligned}
$$

où $s \mapsto R_{U}^{\infty}(s)$ est holomorphe dans le demi-plan $\left\{\sigma=\operatorname{Re}(s)>\alpha^{\prime}\right\}$ et y vérifie $R_{U}^{\infty}(s) \ll_{\sigma} 1+|\tau|$. En utilisant les majorations classiques

$$
\forall \sigma \in \mathbb{R}, \forall \varepsilon>0, \zeta(s) \ll_{\sigma, \varepsilon} 1+|\tau|^{1-\sigma+\varepsilon} ; \quad \forall \sigma>1, \frac{1}{\zeta(s)} \ll_{\sigma} 1 ;
$$

où l'on suppose $|\tau| \geq 1$, il est facile de voir, d'après la relation (37), que pour $\sigma>\alpha^{\prime}$ et tout $\varepsilon>0$, on a

$$
\begin{aligned}
Z_{U}^{\infty}(s) & \ll_{\sigma, \varepsilon}|\zeta(2 b s-1) \zeta(3 b s-2)|+\left|R_{U}^{\infty}(s)\right| \\
& \ll_{\sigma, \varepsilon} 1+|\tau|^{5-5 b \sigma+\varepsilon}+|\tau| .
\end{aligned}
$$

En particulier, pour tout $\varepsilon>0$, on a

$$
Z_{U}^{\infty}\left(\alpha^{\prime}+\varepsilon\right) \ll_{\sigma, \varepsilon}|\tau|^{\frac{5}{3}+\varepsilon} \text { et } Z_{U}^{\infty}(\alpha+\varepsilon) \ll_{\sigma, \varepsilon} 1=|\tau|^{0} .
$$

On déduit d'après le thèorème de Phragmén-Lindelof (voir [17, p. 123]), que $Z_{U}^{\infty}(s) \ll_{\sigma, \varepsilon} 1+|\tau|^{k(\sigma)}$ pour $\sigma \in\left[\alpha^{\prime}+\varepsilon, \alpha+\varepsilon\right]$ et $|\tau| \geq 1$, où $k(\sigma)$ est la fonction affine prenant respectivement les valeurs 0 et $\frac{5}{3}+\varepsilon$ en $\alpha+\varepsilon$ et $\alpha^{\prime}+\varepsilon$. Un calcul facile montre alors que $k(\sigma)=5 b(\alpha-\sigma)+O(\varepsilon)$.

BULLETIN DE LA SOCIÉTÉ MATHÉMATIQUE DE FRANCE 
En conclusion on, a montré que pour $|\tau| \geq 1$, on a

$$
\forall \sigma>\alpha^{\prime}, \forall \varepsilon>0, \quad Z_{U}^{\infty}(s) \ll_{\sigma, \varepsilon} 1+|\tau|^{A(\alpha-\sigma)+\varepsilon} \quad \text { où } A=5 b .
$$

D'un autre côté, en utilisant la relation (37) et les développements au voisinage de zéro $\zeta(1+u)=1 / u+\gamma+O(|u|)$ et $\zeta(2+u)=\zeta(2)+\zeta^{\prime}(2) u+O\left(u^{2}\right)$, on obtient facilement que lorsque $s$ tend vers $\alpha$ :

$$
Z_{U}^{\infty}(s)=\frac{96}{b^{2} \pi^{4}(s-\alpha)^{2}}+\frac{96\left((5 \gamma-1) \pi^{2}-30 \zeta^{\prime}(2)\right)}{b \pi^{6}(s-\alpha)}+O(1) .
$$

En appliquant la proposition 3.1 , on obtient que pour tout $\varepsilon>0$ et $B \rightarrow+\infty$,

$$
N_{\infty}(U, B):=N_{\infty}\left(U, \mathcal{L}_{2 b, b}\right)=B^{\alpha} Q(\log (B))+O_{\varepsilon}\left(B^{\alpha-\mathbf{E}(\mu \delta) / \mathbf{E}(\mu \delta+1) \mu+\varepsilon}\right)
$$

où $\delta=\alpha-\alpha^{\prime}=1 /(3 b), \mu=\sup (1 / \delta, A)=\sup (3 b, 5 b)=5 b$ et où

$$
Q(X)=\mathrm{e}^{-\alpha X} \operatorname{Res}_{s=\alpha}\left(s^{-1} Z_{U}^{\infty}(s) \mathrm{e}^{s X}\right) .
$$

Un calcul facile utilisant la relation (39), donne

$$
Q(X)=\frac{96}{b \pi^{4}} X+\frac{96}{\pi^{6}}\left((5 \gamma-2) \pi^{2}-30 \zeta^{\prime}(2)\right) .
$$

On en déduit que, pour tout $\varepsilon>0$ et $B \rightarrow+\infty$,

$$
\begin{aligned}
N_{\infty}(U, B)=B^{\alpha}\left(\frac{96}{b \pi^{4}} \log (B)+\frac{96}{\pi^{6}}\left((5 \gamma-2) \pi^{2}\right.\right. & \left.\left.-30 \zeta^{\prime}(2)\right)\right) \\
& +O_{\varepsilon}\left(B^{\alpha-1 /(10 b)+\varepsilon}\right) .
\end{aligned}
$$

En conclusion dans le cas $a=2 b>0$ et $i=\infty$, les relations (38) et (39) démontrent les parties 2 et 3 du théorème 2.6 et la relation (40) démontre le corollaire 2.7 .

\section{BIBLIOGRAPHIE}

[1] Batyrev (V.V.) \& Manin (Yu.I.) - Sur le nombre des points rationnels de hauteur bornée des variétés algébriques, Math. Ann., t. 286 (1990), pp. 27-43.

[2] Batyrev (V.V.) \& Tschinkel (Yu.) - Rational points of bounded height on compactifications of anisotropic tori, Internat. Math. Res. Notices, t. 12 (1995), pp. 591-635.

[3] _ Manin's conjecture for Toric variety, J. Algebraic Geom., t. 7 (1998), no. 1, pp. 15-53.

[4] DE LA BRetèche (R.) - Compter des points d'une variété torique, J. Number Theory, t. 87 (2001), no. 2, pp. 315-331.

[5] Chambert-Loir (A.) \& Tschinkel (Yu.) - Points of bounded height on equivariant compactifications of vector groups, I, Compositio Math., t. 124 (2000), no. 1, pp. 65-93. 
[6] Essouabri (D.) - Singularités des séries de Dirichlet associées à des polynômes de plusieurs variables et application à la théorie analytique des nombres, Ann. Inst. Fourier (Grenoble), t. 47 (1997), no. 2, pp. 429-484.

[7] Franke (J.), Manin (Yu.I.) \& Tschinkel (Yu.) - Rational points of bounded height on Fano varieties, Invent. Math., t. 95 (1989), pp. 421-435.

[8] Hardy \& Wright - The theory of numbers, 4 e éd., Clarendon Press, Oxford, 1960.

[9] Landau (E.) - Über einen Satz von Tschebeyschef, Math. Ann., t.61 (1905), pp. 527-550.

[10] _ Über die Anzahl der Gitterpunkte in gewiss en Bereichen (Zweite Abhandlung), Kgl. Ges. d. Wiss. Nachrichten. Math. Phys. Klasse. (Göttingen), t. 2 (1915), pp. 209-243.

[11] Lichtin (B.) - Geometric features of lattice point problems, in Singularity theory (Trieste, 1991), World Sci. Publishing, River Edge, NJ, 1995, pp. 370-443.

[12] Mahler (K.) - Über einer Satz von Mellin, Math. Ann., t. 100 (1928), pp. 384-398.

[13] Peyre (E.) - Étude asymptotique des points de hauteur bornée, École d'été sur la géométrie des variétés toriques, Grenoble.

[14] _ Hauteurs et mesures de Tamagawa sur les variétés de Fano, Duke Math. J., t. 79 (1995), pp. 101-218.

[15] Schanuel (S.H.) - Heights in number fields, Bull. Soc. math. France, t. 107 (1979), pp. 433-449.

[16] Serre (J.-P.) - Lectures on the Mordell-Weil theorem, $2^{\mathrm{e}}$ éd., Freidr. Vieweg \& Sohn, Braunschweig/Wiesbaden, 1990.

[17] Tenenbaum (G.) - Introduction à la théorie analytique et probabiliste des nombres, Cours Spécialisés, vol. 1, Société Mathématique de France, Paris, 1995. 